\title{
Strong Convergence of the Split-Step $\theta$-Method for Stochastic Age-Dependent Capital System with Random Jump Magnitudes
}

\author{
Jianguo Tan, ${ }^{1}$ A. Rathinasamy, ${ }^{2}$ Hongli Wang, ${ }^{3}$ and Yongfeng Guo ${ }^{1}$ \\ ${ }^{1}$ Department of Mathematics, Tianjin Polytechnic University, Tianjin 300387, China \\ ${ }^{2}$ Department of Mathematics, PSG College of Technology, Coimbatore 641004, India \\ ${ }^{3}$ Department of Mechanics, Tianjin University, Tianjin 300072, China
}

Correspondence should be addressed to Jianguo Tan; tanjg@tju.edu.cn

Received 26 February 2014; Accepted 20 March 2014; Published 15 April 2014

Academic Editor: Jifeng Chu

Copyright (C) 2014 Jianguo Tan et al. This is an open access article distributed under the Creative Commons Attribution License, which permits unrestricted use, distribution, and reproduction in any medium, provided the original work is properly cited.

\begin{abstract}
We develop a new split-step $\theta$ (SS $\theta$ ) method for stochastic age-dependent capital system with random jump magnitudes. The main aim of this paper is to investigate the convergence of the SS $\theta$ method for a class of stochastic age-dependent capital system with random jump magnitudes. It is proved that the proposed method is convergent with strong order $1 / 2$ under given conditions. Finally, an example is simulated to verify the results obtained from theory.
\end{abstract}

\section{Introduction}

Stochastic partial differential equations are becoming increasingly used to model real-world phenomena in different fields, such as economics, biology, and physics. Recently, the study of the stochastic age-dependent (vintage) capital system has received a great deal of attention. For example, Wang studied stability of solutions for stochastic investment system [1]. Zhang et al. studied the convergence and exponential stability of numerical solutions to the stochastic age-dependent capital system $[2,3]$.

In the stochastic age-dependent capital system, due to the effects of external environment for capital system, such as innovations in technique, introduction of new products, natural disasters, and changes in laws and government policies, the size of the capital systems increases or decreases drastically. So Poisson jumps with deterministic jump magnitude have been used in stochastic age-dependent population equations. For example, Li et al. [4] studied the Euler numerical method for stochastic age-dependent population equations with Poisson jumps. L. Wang and X. Wang [5] analysed the convergence of the semi-implicit Euler method for stochastic age-dependent population equations with Poisson jumps. Rathinasamy et al. [6] developed the numerical method for stochastic age-dependent population equations with Poisson jump and phase semi-Markovian switching. However, the random jump magnitude is now commonly seen in financial models [7-9]. In this paper, we will consider the following stochastic age-dependent capital system with random jump magnitudes as shown in [10]:

$$
\begin{aligned}
& \mathrm{d} K(t, a)=-\mu(t, a) K(t, a) \mathrm{d} t \\
&+f(t, K(t, a)) \mathrm{d} t+g(t, K(t, a)) \mathrm{d} W(t) \\
&+h\left(t, K(t, a), \gamma_{N(t)+1}\right) \mathrm{d} N(t), \quad \text { in } D, \\
& K(t, 0)= \varphi(t) \\
&= \alpha(t) B(t) F\left(L(t), \int_{0}^{A} K(t, a) \mathrm{d} a\right), \\
& \text { in } t \in[0, T], \\
& K(0, a)=K_{0}(a), \quad \text { in } a \in[0, A], \\
& \mathbb{N}(t)=\int_{0}^{A} K(t, a) \mathrm{d} a, \quad \text { in } t \in[0, T],
\end{aligned}
$$

where $K(t, a)$ denotes the stock of capital goods of age $a$ at time $t, \mathrm{~d} K(t, a)=(\partial K(t, a) / \partial t+\partial K(t, a) / \partial a) \mathrm{d} t, \varphi(0)=$ $K_{0}(0)$, and $D=[0, T] \times[0, A] \cdot \mathbb{N}(t)$ is defined as total 
output produced in year $t$; also $a$ is the age of the capital; the investment $\varphi(t)$ in the new capital. The $f$ is the appreciation (when $f \geq 0$ ) or depreciation (when $f \leq 0$ ) of the production capacity, and $g$ represents the volatility of the capital stock. The value of $h$ is the actual jump and $\gamma_{i}$ is the underlying random variables of the magnitudes, and often it is called "mark" of the jump.

Also $W(t)$ is a standard Wiener process. $N(t)$ is a scalar Poisson process with intensity $\lambda_{1}$. It is assumed that for some $q \geq 1$ there is a constant $C$ such that $\mathbb{E}\left[\left|\gamma_{i}\right|^{2 q}\right] \leq C$; that is, the $2 q$ th moment of the jump magnitude is bounded. The maximum physical lifetime of capital $A$, the planning interval of calendar time $[0, T)$, the depreciation rate $\mu(t, a)$ of capital, and the capital density $K_{0}(a)$ (the initial distribution of capital over age)are given. The $\alpha(t)$ denotes the accumulative rate of capital at the moment of $t, 0<\alpha(t)<1$, and $B(t)$ is the technical progress at the moment of $t$. This makes that total output produced in year $t$ be defined as $\mathbb{N}(t)=\int_{0}^{A} K(t, a) \mathrm{d} a$. In each sector all the firms have an identical neoclassical technology and produce output using labor and capital. The production function $F\left(L(t), \int_{0}^{A} K(t, a) \mathrm{d} a\right)$ is neoclassical, where $\int_{0}^{A} K(t, a) \mathrm{d} a$ is the total sum of capital at time $t$ and $L(t)$ is the labor force.

The integral version of (1) is given by the equation

$$
\begin{aligned}
K_{t}= & K_{0}-\int_{0}^{t} \frac{\partial K_{s}}{\partial a} \mathrm{~d} s-\int_{0}^{t} \mu(s, a) K_{s} \mathrm{~d} s+\int_{0}^{t} f\left(s, K_{s}\right) \mathrm{d} s \\
& +\int_{0}^{t} g\left(s, K_{s}\right) \mathrm{d} W(s)+\int_{0}^{t} h\left(s, K_{s}, \gamma(s)\right) \mathrm{d} N(s),
\end{aligned}
$$

where $K_{t}=K(t, a)$ for fixed $a$.

Since the system (1) does not have closed form solutions, it is necessary to develop numerical methods for (1). Recently, Zhang and Rathinasamy [10] first derived the numerical solutions for stochastic age-dependent capital system with random jump magnitudes. However, their method belongs to the classic explicit Euler method and has a lower accuracy in [10] if we do not consider the appropriate step sizes.

Higham and Kloeden [11] first constructed the splitstep backward Euler (SSBE) method for nonlinear stochastic differential equations with Poisson jumps. Tan and Wang [12] studied the convergence and stability of the SSBE method for linear stochastic delay integrodifferential equations. Ding et al. [13] developed the split-step $\theta$ method for solving the stochastic differential equations. Rathinasamy [14] investigated the split-step $\theta$ methods for stochastic age-dependent population equations with Markovian switching. Thus, we can construct the SS $\theta$ method for stochastic age-dependent population equations with random Poisson jumps.

In this paper, we will investigate the convergence of the $\operatorname{SS} \theta$ method for system (1). The outline of the paper is as follows. In Section 2, we will introduce some preliminary results which are essential for our analysis. Section 3 will show us the $\mathrm{SS} \theta$ method for solving stochastic age-dependent population equations with random Poisson jumps. In Section 4, several lemmas which are useful for our main result are proved. We give the main result that the numerical solutions converge to the true solutions with strong order $1 / 2$ in Section 5. At last, a numerical example is given to verify the results obtained from the theory.

\section{Preliminaries}

Throughout this paper, it will be denoted by $L^{2}([0, A])$ the space of functions that are square-integrable over the domain $[0, A]$. Let

$$
V=H^{1}([0, A]) \equiv\left\{\xi \mid \xi \in L^{2}([0, A]), \frac{\partial \xi}{\partial a} \in L^{2}([0, A])\right\},
$$

where $\partial \xi / \partial a$ is generalized partial derivative with respect to age $a$ and $V$ is a Sobolev space. $H=L^{2}([0, A])$ such that $V \hookrightarrow$ $H \equiv H^{\prime} \hookrightarrow V^{\prime} . V^{\prime}=H^{-1}([0, A])$ is the dual space of $V$. We denote by $\|\cdot\|,|\cdot|$ and $\|\cdot\|_{*}$ the norms in $V, H$, and $V^{\prime}$, respectively, by $(\cdot, \cdot)$ the scalar product in $H .\langle\cdot, \cdot\rangle$, the duality product between $V$ and $V^{\prime}$, is defined by

$$
\langle\cdot, \cdot\rangle=\int_{0}^{A} u \cdot v \mathrm{~d} a, \quad u \in V, v \in V^{\prime}
$$

Let $W(t)$ be a Wiener process defined on $(\Omega, \mathscr{F}, \mathbb{P})$ and taking its values in the separable Hilbert space $S$,

$$
W(t)=\sum_{i=1}^{\infty} \beta_{i}(t) e_{i}
$$

where $\left\{e_{i}\right\}_{i \geq 0}$ is an orthonormal set of eigenvectors of $G$, $\beta_{i}(t)$ are mutually independent real Wiener processes with incremental covariance $\lambda_{i}>0, G e_{i}=\lambda_{i} e_{i}$, and $\operatorname{tr} G=\sum_{i=1}^{\infty} \lambda_{i}$ (tr denotes the trace of an operator). For an operator $g \in$ $\mathscr{L}(S, H)$ to be the space of all bounded linear operators from $S$ into $H$, it is denoted by $\|g\|_{2}$ the Hilbert-Schmidt norm; that is,

$$
\|g\|_{2}^{2}=\operatorname{tr}\left(g G g^{T}\right)
$$

Let $C=C([0, T] ; H)$ be the space of all continuous function from $[0, T]$ into $H$ with sup-norm $\|\phi\|_{c}=\sup _{0 \leq s \leq T}|\phi|(s)$, $L_{V}^{p}=L^{p}([0, T] ; V)$, and $L_{H}^{p}=L^{p}([0, T] ; H)$.

Let $(\Omega, \mathscr{F}, \mathbb{P})$ be a complete probability space with a filtration $\left\{\mathscr{F}_{t}\right\}_{t \geq 0}$, satisfying the usual conditions (i.e., it is increasing and right continuous while $\mathscr{F}_{0}$ contains all $\mathbb{P}$-null sets).

Definition 1. Let $\left(\Omega, \mathscr{F},\left\{\mathscr{F}_{t}\right\}, \mathbb{P}\right)$ be the stochastic basis and $W_{t}$ a Wiener process. Suppose that $K_{0}$ is a random variable such that $\mathbb{E}\left|K_{0}\right|^{2}<\infty$. A stochastic process $K_{t} \equiv K(t, a)$ for fixed $a$ is said to be a solution on $\Omega$ to the stochastic age-structured capital system for $t \in[0, T]$ if the following conditions are satisfied:

(D1) $K_{t}$ is a $\mathscr{F}_{t}$-measurable random variable;

(D2) $K_{t} \in I^{p}([0, T] ; V) \cap L^{2}(\Omega ; C([0, T] ; V)), p>1, T>0$, where $I^{p}([0, T] ; V)$ denotes the space of all $V$-valued processes $\left(K_{t}\right)_{t \in[0, T]}$ (one will write $K_{t}$ for short) 
measurable (from $[0, T] \times \Omega$ into $V$ ), and satisfying $\mathbb{E} \int_{0}^{T}\left\|K_{t}\right\|^{p} \mathrm{~d} t<\infty$. Here $C([0, T] ; V)$ denotes the space of all continuous functions from $[0, T]$ to $V$;

(D3) it satisfies the following equation:

$$
\begin{aligned}
&\left\langle K_{t}, v\right\rangle+\int_{0}^{t}\left\langle\frac{\partial K_{s}}{\partial a}, v\right\rangle \mathrm{d} s \\
&=\left\langle K_{0}, v\right\rangle-\int_{0}^{t}\left\langle\mu(s, a) K_{s}, v\right\rangle \mathrm{d} s \\
&+\int_{0}^{t}\left\langle f\left(s, K_{s}\right), v\right\rangle \mathrm{d} s \\
&+\int_{0}^{t}\left\langle g\left(s, K_{s}\right), v\right\rangle \mathrm{d} W(s) \\
&+\int_{0}^{t}\left\langle h\left(s, K_{s}, \gamma_{N(s)+1}\right), v\right\rangle \mathrm{d} N(s),
\end{aligned}
$$

for all $v \in V, t \in[0, T]$, a.e. $\omega \in \Omega$, where the stochastic integrals are understood in the Itô sense.

The parameter $A$ is the maximal age of the capital, so $K(t, a) \equiv K_{t}=0, \forall a \geq A$.

As the standing hypotheses we always assume that the following conditions are satisfied.

$(\mathbb{M 1}) f(t, 0)=0, g(t, 0)=0$, and $h\left(t, 0, \gamma_{N(t)+1}\right)=0, t \in$ $[0, T]$.

([2) (Lipschitz condition) there exists a positive constant $k$ such that $x, y \in H$ and $\forall t$,

(i) $|f(t, y)-f(t, x)| \vee\|g(t, y)-g(t, x)\|_{2} \leq k|y-x|$, (ii) $|h(t, y, u)-h(t, x, v)| \leq k[|y-x|+|u-v|]$.

$(\mathbb{H} 3) \mu(t, a)$ is nonnegative measurable in $D$ such that

$$
0 \leq \mu_{0} \leq \mu(t, a) \leq \bar{\mu}<\infty,
$$

and $B(t)$ is nonnegative continuous in $[0, T]$ such that

$\alpha(t) B(t) \leq \eta ; \quad \eta \quad$ is a non-negativeconstant in $[0, T]$.

$(\mathbb{H} 4) F(L, N) \geq 0,(F(L, 0)=0), \partial F / \partial L>0,0<\partial F / \partial N \leq$ $F_{1}$, where $F_{1}$ is a positive constant.

In an analogous way to the corresponding proof presented in [15], the following existence and uniqueness of solutions is established: under the conditions $(\mathbb{M} 1)-(\mathbb{4} 4),(1)$ has a unique continuous solution $K(t, a)$ on $(t, a) \in D$.

We note for the following jump process:

$$
\gamma(t):=\gamma_{N(t)+1}=\sum_{j} \gamma_{j+1} I_{\left[\tau_{j}, \tau_{j+1}\right)}(t),
$$

where $\tau_{0}=0$ and $I_{A}$ is the indicator function for the set $A$; that is, $I_{A}(x)=\left\{\begin{array}{l}1, x \in A \\ 0, x \notin A\end{array}\right.$.

Then the following lemma can be found in $[16,17]$.
Lemma 2. There exists a constant $B$ for any $t \in[0, T]$ and $\mathbb{E}\left[\left|\gamma_{i}\right|^{2 q}\right] \leq B$ for some $q>1$ such that

$$
\mathbb{E}\left[\int_{0}^{t}|\bar{\gamma}(s)-\gamma(s)|^{2} d s\right] \leq C \Delta^{1-(1 / q)}
$$

\section{The Split-Step $\theta$-Method}

Let $\tau_{j}$ denote the $j$ th jump of $N(s)$ occurrence time. Suppose, for example, that jumps arrive at distinct, ordered times $\tau_{1}<\tau_{2}<, \ldots$; let $t_{1}, t_{2}, \ldots, t_{m}$ be a deterministic grid point of $[0, T]$. We construct approximate solutions to models of the form (1) at a discrete set of times $\tau_{n}$. This set is the superposition of the random jump times of a Poisson process on $[0, T]$ and a deterministic grid $t_{1}, t_{2}, \ldots, t_{m}$ and satisfies $\max \left|\tau_{i+1}-\tau_{i}\right|<\Delta$. Let $\Delta=t_{n+1}-t_{n}, \Delta W_{n}:=W\left(t_{n+1}\right)-$ $W\left(t_{n}\right)$, and $\Delta N_{n}:=N\left(t_{n+1}\right)-N\left(t_{n}\right)$ denote the increments of the time, Brownian motion, and the Poisson processes, respectively.

For system (1) the split-step $\theta$ approximate solution is defined by the iterative scheme

$$
\begin{array}{r}
Q_{n}^{*}=Q_{n}-\frac{\partial Q_{n+1}}{\partial a}+\left[(1-\theta)\left[-\mu\left(t_{n}, a\right) Q_{n}+f\left(t_{n}, Q_{n}\right)\right]\right. \\
\left.+\theta\left[-\mu\left(t_{n}, a\right) Q_{n}^{*}+f\left(t_{n}, Q_{n}^{*}\right)\right]\right] \Delta \\
Q_{n+1}=Q_{n}^{*}+g\left(t_{n}, Q_{n}^{*}\right) \Delta W_{n}+h\left(t_{n}, Q_{n}^{*}, \gamma_{N\left(t_{n}\right)+1}\right) \Delta N_{n}
\end{array}
$$

with initial values $Q_{0}=K(0, A), Q_{n}(t, 0)=\int_{0}^{A} \beta(t, a) Q_{n} \mathrm{~d} a$, $n>1 ; Q_{n}$ is the numerical approximation of $K\left(t_{n}, a\right)$ with $t_{n}=n \cdot \Delta$; the time increment is $\Delta=T / N \ll 1$. Where $\theta \in[0,1]$, if we give $\theta=1$, the SS $\theta$ method becomes the SSBE method in [11]. If $\theta=0$, the $\operatorname{SS} \theta$ method is an explicit method.

To answer the question of the existence of numerical solution, we will give the following lemma.

Lemma 3. Let conditions $(\mathbb{H} 2)$ and $(\mathbb{H} 3)$ hold, and let $0<\theta<$ 1 and $0<\Delta<1 / \theta(k+\bar{\mu})$; then the implicit equation (12a) can be solved uniquely for $Q_{n}^{*}$, with probability 1 .

Proof. Writing (12a) as $Q_{n}^{*}=F\left(Q_{n}^{*}\right)=y+\theta \Delta\left[-\mu\left(t_{n}, a\right) Q_{n}^{*}+\right.$ $\left.f\left(t_{n}, Q_{n}^{*}\right)\right]$, and using condition $(\mathbb{2} 2)$ and $(\mathbb{Z} 3)$, we have

$$
\begin{aligned}
|F(u)-F(v)| & \leq \theta \Delta[|f(t, u)-f(t, v)|+|\mu(t, a)(u-v)|] \\
& \leq \theta \Delta(k+\bar{\mu})|u-v|
\end{aligned}
$$

Then the result follows from the classical Banach contraction mapping theorem [18].

When Lemma 3 followed, we find it is convenient to use continuous-time approximation solution in our strong 
convergence analysis; hence for $\in\left[t_{n}, t_{n+1}\right)$, we can define the following step functions:

$$
\begin{gathered}
Z_{1}(t)=Z_{1}(t, a)=\sum_{n=0}^{N-1} Q_{n} I_{[n \Delta t,(n+1) \Delta t)}(t), \\
Z_{2}(t)=Z_{2}(t, a) \sum_{n=0}^{N-1} Q_{n}^{*} I_{[n \Delta t,(n+1) \Delta t)}(t), \\
\bar{\gamma}(t)=\sum_{n=0}^{N-1} \gamma\left(t_{n}\right) I_{[n \Delta,(n+1) \Delta)}(t),
\end{gathered}
$$

where $N$ is the largest number such that $N \Delta \leq T$.

When $t \in\left[t_{n}, t_{n+1}\right)$, Lemma 3 ensures the existence of $Q_{n}^{*}$ by (12a); then we define

$$
\begin{aligned}
Q_{t}= & Q_{0}-\int_{0}^{t} \frac{\partial Q_{s}}{\partial a} \mathrm{~d} s \\
& +\int_{0}^{t}(1-\theta)\left[-\mu(s, a) Z_{1}(s)+f\left(s, Z_{1}(s)\right)\right] \mathrm{d} s \\
& +\int_{0}^{t} \theta\left[-\mu(s, a) Z_{2}(s)+f\left(s, Z_{2}(s)\right)\right] \mathrm{d} s \\
& +\int_{0}^{t} g\left(s, Z_{2}(s)\right) \mathrm{d} W_{s}+\int_{0}^{t} h\left(s, Z_{2}(s), \bar{\gamma}(s)\right) \mathrm{d} N_{s},
\end{aligned}
$$

with initial value $Q_{0}=p(0, a), Q(t, 0)=\int_{0}^{A} \beta(t, a) Q_{t} \mathrm{~d} a, Q_{t}=$ $Q(t, a)$.

It is easy to verify that $Z_{1}\left(t_{n}, a\right)=Q_{n}=Q\left(t_{n}, a\right)$; that is, $Z_{1}(t, a)$ and $Q(t, a)$ coincide with the discrete solutions at the grid points. Hence we refer to $Q(t, a)$ as a continuous-time extension of the discrete approximation $\left\{Q_{n}\right\}$. So our plan is to prove a strong convergence result for $Q(t, a)$.

\section{Several Lemmas}

In this section, we will give several lemmas which are useful for the following main result.

Lemma 4. Under the conditions $(\mathbb{H} 1)-(\mathbb{4} 4)$, there are constants $p \geq 2$ and $C_{1}>0$ such that

$$
\mathbb{E}\left[\sup _{0 \leq t \leq T}\left|K_{t}\right|^{p}\right] \leq C_{1} .
$$

The proof is similar to that in [3].

Next lemma shows the relationship between $\mathbb{E}\left|Q_{n}^{*}\right|^{2}$ and $\mathbb{E}\left|Q_{n}\right|^{2}$.

Lemma 5. Under conditions $(\mathbb{H} 1)-(\mathbb{H} 4)$, let $0<\theta<1,0<$ $\Delta<\min \left\{1,1 / \theta(k+\bar{\mu}), 1 / 2 \sqrt{3\left(\bar{\mu}^{2}+k^{2}\right)}\right\}$, and $\mathbb{E}\left|\partial Q_{n+1} / \partial a\right|^{2}<$ $\infty$; then there exist two positive constants $C_{2}$ and $C_{3}$ such that

$$
\mathbb{E}\left|Q_{n}^{*}\right|^{2} \leq C_{2} \mathbb{E}\left|Q_{n}\right|^{2}+C_{3},
$$

where $Q_{n}^{*}$ and $Q_{n}$ are produced by (12a) and (12b).
Proof. Squaring both sides of (12a) and using the elementary inequality $(a+b+c)^{2} \leq 3 a^{2}+3 b^{2}+3 c^{2}$, we find

$$
\begin{aligned}
&\left|Q_{n}^{*}\right|^{2} \leq 3\left|Q_{n}\right|^{2}+3\left|\frac{\partial Q_{n+1}}{\partial a}\right|^{2} \Delta^{2} \\
&+3\left[(1-\theta)\left[-\mu\left(t_{n}, a\right) Q_{n}+f\left(t_{n}, Q_{n}\right)\right]\right. \\
&\left.+\theta\left[-\mu\left(t_{n}, a\right) Q_{n}^{*}+f\left(t_{n}, Q_{n}^{*}\right)\right]\right]^{2} \Delta^{2} .
\end{aligned}
$$

Using the elementary inequality $|(1-\theta) x+\theta y|^{2} \leq(1-\theta)|x|^{2}+$ $\theta|y|^{2}$, we obtain

$$
\begin{aligned}
\left|Q_{n}^{*}\right|^{2} \leq & 3\left|Q_{n}\right|^{2}+3\left|\frac{\partial Q_{n+1}}{\partial a}\right|^{2} \Delta^{2} \\
& +3(1-\theta)\left[-\mu\left(t_{n}, a\right) Q_{n}+f\left(t_{n}, Q_{n}\right)\right]^{2} \Delta^{2} \\
& +3 \theta\left[-\mu\left(t_{n}, a\right) Q_{n}^{*}+f\left(t_{n}, Q_{n}^{*}\right)\right]^{2} \Delta^{2} .
\end{aligned}
$$

Due to $0<\theta<1$ and conditions $(\mathbb{\square} 2)-(\mathbb{M} 3)$, we can get

$$
\begin{aligned}
\left|Q_{n}^{*}\right|^{2} \leq & 3\left|Q_{n}\right|^{2}+3\left|\frac{\partial Q_{n+1}}{\partial a}\right|^{2} \Delta^{2} \\
& +6\left[\left|\mu\left(t_{n}, a\right) Q_{n}\right|^{2}+\left|f\left(t_{n}, Q_{n}\right)\right|^{2}\right] \Delta^{2} \\
& +6\left[\left|\mu\left(t_{n}, a\right) Q_{n}^{*}\right|^{2}+\left|f\left(t_{n}, Q_{n}^{*}\right)\right|^{2}\right] \Delta^{2} \\
\leq & 3\left|Q_{n}\right|^{2}+3\left|\frac{\partial Q_{n+1}}{\partial a}\right|^{2} \Delta^{2}+6\left(\bar{\alpha}^{2}+k^{2}\right)\left|Q_{n}\right|^{2} \Delta^{2} \\
& \left.+6\left(\bar{\alpha}^{2}+k^{2}\right)\left|Q_{n}^{*}\right|^{2}\right] \Delta^{2} .
\end{aligned}
$$

Taking mathematical expectation for both sides, we can obtain

$$
\begin{aligned}
\mathbb{E}\left|Q_{n}^{*}\right|^{2} \leq & 3 \mathbb{E}\left|\frac{\partial Q_{n+1}}{\partial a}\right|^{2} \Delta^{2}+\left[3+6\left(\bar{\alpha}^{2}+k^{2}\right) \Delta^{2}\right] \mathbb{E}\left|Q_{n}\right|^{2} \\
& +6\left(\bar{\alpha}^{2}+k^{2}\right) \Delta^{2} \mathbb{E}\left|Q_{n}^{*}\right|^{2} .
\end{aligned}
$$

Since $6\left(\bar{\alpha}^{2}+k^{2}\right) \Delta^{2}<1 / 2$, thus $1-6\left(\bar{\alpha}^{2}+k^{2}\right) \Delta^{2} \geq 1 / 2$; then by $0<\Delta<1$, we have

$$
\begin{aligned}
\mathbb{E}\left|Q_{n}^{*}\right|^{2} & \leq \frac{3+6\left(\bar{\alpha}^{2}+k^{2}\right) \Delta^{2}}{1-6\left(\bar{\alpha}^{2}+k^{2}\right) \Delta^{2}} \mathbb{E}\left|Q_{n}\right|^{2}+\frac{3 \mathbb{E}\left|\partial Q_{n+1} / \partial a\right|^{2} \Delta^{2}}{1-6\left(\bar{\alpha}^{2}+k^{2}\right) \Delta^{2}} \\
& \leq 6\left[1+2\left(\bar{\alpha}^{2}+k^{2}\right)\right] \mathbb{E}\left|Q_{n}\right|^{2}+6 \mathbb{E}\left|\frac{\partial Q_{n+1}}{\partial a}\right|^{2} \\
& =C_{2} \mathbb{E}\left|Q_{n}\right|^{2}+C_{3},
\end{aligned}
$$

where $C_{2}=6+12\left(\bar{\alpha}^{2}+k^{2}\right)$ and $C_{3}=6 \mathbb{E}\left|\partial Q_{n+1} / \partial a\right|^{2}$. The proof is completed.

The next lemma shows that the continuous numerical solutions $Q_{t}$ have bounded second moments. 


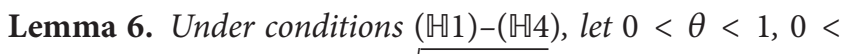
$\Delta<\min \left\{1,1 / \theta(k+\bar{\mu}), 1 / 2 \sqrt{3\left(\bar{\mu}^{2}+k^{2}\right)}\right\}$; then there exists a constant $C_{4}>0$ such that

$$
\mathbb{E}\left[\sup _{t \in[0, T]}\left|Q_{t \wedge v_{n}}\right|^{2}\right] \leq C_{4},
$$

where $\tau_{n}:=\inf \left\{t \geq 0,\left|K_{t}\right| \geq n\right\}, \sigma_{n}:=\inf \left\{t \geq 0,\left|Q_{t}\right| \geq n\right\}$, and $v_{n}=\tau_{n} \wedge \sigma_{n}$.

Proof. From (17), we can get

$$
\begin{aligned}
\mathrm{d} Q_{t}= & -\frac{\partial Q_{t}}{\partial a} \mathrm{~d} t+(1-\theta)\left[-\mu(t, a) Z_{1}(t)+f\left(s, Z_{1}(t)\right)\right] \mathrm{d} t \\
& +\theta\left[-\mu(t, a) Z_{2}(t)+f\left(s, Z_{2}(t)\right)\right] \mathrm{d} t \\
& +g\left(s, Z_{2}(t)\right) \mathrm{d} W_{t}+h\left(s, Z_{2}(t), \bar{\gamma}(t)\right) \mathrm{d} N_{t} .
\end{aligned}
$$

Applying the Itô formula [19] to $\left|Q_{t \wedge v_{n}}\right|^{2}$ it yields

$$
\begin{aligned}
\left|Q_{t \wedge v_{n}}\right|^{2}= & \left|Q_{0}\right|^{2}+2 \int_{0}^{t \wedge v_{n}}\left\langle-\frac{\partial Q_{s}}{\partial a}, Q_{s}\right\rangle \mathrm{d} s \\
& -2 \int_{0}^{t \wedge v_{n}}\left((1-\theta) \mu(s, a) Z_{1}(s)\right. \\
& +2 \int_{0}^{t \wedge v_{n}}\left((1-\theta) f\left(s, Z_{1}(s)\right)\right. \\
& +2 \int_{0}^{t \wedge v_{n}}\left(Q_{s}, g\left(s, Z_{s}\right)\right) \mathrm{d} W(s) \\
& +2 \int_{0}^{t \wedge v_{n}}\left(Q_{s}, h\left(s, Z_{s}, \bar{\gamma}(s)\right)\right) \mathrm{d} N(s) \\
& +\int_{0}^{t \wedge v_{n}}\left\|g\left(s, Z_{s}\right)\right\|_{2}^{2} \mathrm{~d} s \\
& +\int_{0}^{t \wedge v_{n}}\left|h\left(s, Z_{s}, \bar{\gamma}(s)\right)\right|^{2} \mathrm{~d} N(s) .
\end{aligned}
$$

Using conditions $(\mathbb{M} 1)-(\mathbb{Q}-3)$ and the compensated Poisson process $\widetilde{N}(t):=N(t)-\lambda_{1} t$, we have

$$
\begin{aligned}
&\left|Q_{t \wedge v_{n}}\right|^{2} \leq\left|Q_{0}\right|^{2}+2 \int_{0}^{t \wedge v_{n}}\left\langle-\frac{\partial Q_{s}}{\partial a}, Q_{s}\right\rangle \mathrm{d} s \\
&+2 \mu_{0} \int_{0}^{t \wedge v_{n}}\left|Q_{s}\right|\left|(1-\theta) Z_{1}(s)+\theta Z_{2}(s)\right| \mathrm{d} s \\
&+2 \int_{0}^{t \wedge v_{n}}\left|Q_{s}\right| \mid(1-\theta) f\left(s, Z_{1}(s)\right) \\
&+\theta f\left(s, Z_{2}(s)\right) \mid \mathrm{d} s
\end{aligned}
$$

$$
\begin{aligned}
& +2 \int_{0}^{t \wedge v_{n}}\left(Q_{s}, g\left(s, Z_{2}(s)\right) \mathrm{d} W_{s}\right) \\
& +\int_{0}^{t \wedge v_{n}}\left\|g\left(s, Z_{2}(s)\right)\right\|_{2}^{2} \mathrm{~d} s \\
& +2 \int_{0}^{t \wedge v_{n}}\left(Q_{s}, h\left(s, Z_{2}(s), \bar{\gamma}(s)\right)\right) \mathrm{d} \widetilde{N}_{s} \\
& +\int_{0}^{t \wedge v_{n}}\left|h\left(s, Z_{2}(s), \bar{\gamma}(s)\right)\right|^{2} \mathrm{~d} \widetilde{N}_{s} \\
& +\lambda_{1} \int_{0}^{t \wedge v_{n}}\left|h\left(s, Z_{2}(s), \bar{\gamma}(s)\right)\right|^{2} \mathrm{~d} s \\
& +2 \lambda_{1} \int_{0}^{t \wedge v_{n}}\left|Q_{s}\right|\left|h\left(s, Z_{2}(s), \bar{\gamma}(s)\right)\right| \mathrm{d} s
\end{aligned}
$$

Note

$$
\begin{aligned}
\left\langle-\frac{\partial Q_{s}}{\partial a}, Q_{s}\right\rangle & =-\int_{0}^{A} Q_{s} \cdot \frac{\partial Q_{s}}{\partial a} \mathrm{~d} a \\
= & \frac{1}{2} \alpha^{2}(s) A^{2}(s)\left[F\left(L(s), \int_{0}^{A} Q_{s} \mathrm{~d} a\right)\right. \\
& -F(L(s), 0)]^{2} \\
\leq & \frac{1}{2} \eta^{2}\left(\left.\frac{\partial F(L, N)}{\partial N}\right|_{y}\right)^{2}\left(\int_{0}^{A} Q_{s} \mathrm{~d} a\right) \\
\leq & \frac{1}{2} A F_{1}^{2} \eta^{2}\left|Q_{s}\right|^{2},
\end{aligned}
$$

where $y \in\left(0, \int_{0}^{A} Q_{s} \mathrm{~d} a\right)$. Consider

$$
\begin{aligned}
& 2 \mu_{0} \int_{0}^{t \wedge v_{n}}\left|Q_{s}\right|\left|(1-\theta) Z_{1}(s)+\theta Z_{2}(s)\right| \mathrm{d} s \\
& \quad \leq \mu_{0} \int_{0}^{t \wedge v_{n}}\left|Q_{s}\right|^{2} \mathrm{~d} s+2 \mu_{0} \int_{0}^{t \wedge v_{n}}\left[\left|Z_{1}(s)\right|^{2}+\left|Z_{2}(s)\right|^{2}\right] \mathrm{d} s,
\end{aligned}
$$

$2 \int_{0}^{t \wedge v_{n}}\left|Q_{s}\right|\left|(1-\theta) f\left(s, Z_{1}(s)\right)+\theta f\left(s, Z_{2}(s)\right)\right| \mathrm{d} s$

$$
\begin{aligned}
\leq & \int_{0}^{t \wedge v_{n}}\left|Q_{s}\right|^{2} \mathrm{~d} s \\
& +2 \int_{0}^{t \wedge v_{n}}\left[\left|f\left(s, Z_{1}(s)\right)\right|^{2}+\left|f\left(s, Z_{2}(s)\right)\right|^{2}\right] \mathrm{d} s \\
\leq & \int_{0}^{t \wedge v_{n}}\left|Q_{s}\right|^{2} \mathrm{~d} s+2 k^{2} \int_{0}^{t \wedge v_{n}}\left[\left|Z_{1}(s)\right|^{2}+\left|Z_{2}(s)\right|^{2}\right] \mathrm{d} s,
\end{aligned}
$$

$2 \lambda_{1} \int_{0}^{t \wedge v_{n}}\left|Q_{s}\right|\left|h\left(s, Z_{2}(s), \bar{\gamma}(s)\right)\right| \mathrm{d} s$ 


$$
\begin{aligned}
& \leq \lambda_{1} \int_{0}^{t \wedge v_{n}}\left|Q_{s}\right|^{2} \mathrm{~d} s+\lambda_{1} \int_{0}^{t \wedge v_{n}}\left|h\left(s, Z_{2}(s), \bar{\gamma}(s)\right)\right|^{2} \mathrm{~d} s \\
& \leq \lambda_{1} \int_{0}^{t \wedge v_{n}}\left|Q_{s}\right|^{2} \mathrm{~d} s+\lambda_{1} k^{2} \int_{0}^{t \wedge v_{n}}\left|Z_{2}(s)\right|^{2}+|\bar{\gamma}(s)-\gamma(s)|^{2} \mathrm{~d} s .
\end{aligned}
$$

Taking (29)-(30) into (28), we compute that for some positive constants $K_{1}=A^{2} F_{1}^{2} \eta^{2}+1+\mu_{0}+\lambda_{1}, K_{2}=2\left(k^{2}+\mu_{0}\right), K_{3}=$ $3 k^{2}+2 \lambda_{1} k^{2}+2 \mu_{0}$

$$
\begin{aligned}
\left|Q_{t \wedge v_{n}}\right|^{2} \leq & \left|Q_{0}\right|^{2}+K_{1} \int_{0}^{t \wedge v_{n}}\left|Q_{s}\right|^{2} \mathrm{~d} s \\
& +K_{2} \int_{0}^{t \wedge v_{n}}\left|Z_{1}(s)\right|^{2} \mathrm{~d} s+K_{3} \int_{0}^{t \wedge v_{n}}\left|Z_{2}(s)\right|^{2} \mathrm{~d} s \\
& +\lambda_{1} K^{2} \int_{0}^{t \wedge v_{n}}|\bar{\gamma}(s)-\gamma(s)|^{2} \mathrm{~d} s \\
& +2 \int_{0}^{t \wedge v_{n}}\left(Q_{s}, g\left(s, Z_{2}(s)\right) \mathrm{d} W_{s}\right) \\
& +2 \int_{0}^{t \wedge v_{n}}\left(Q_{s}, h\left(s, Z_{2}(s), \bar{\gamma}(s)\right)\right) \mathrm{d} \widetilde{N}_{s} \\
& +\int_{0}^{t \wedge v_{n}}\left|h\left(s, Z_{2}(s), \bar{\gamma}(s)\right)\right|^{2} \mathrm{~d} \widetilde{N}_{s} .
\end{aligned}
$$

Now, it follows that for any $t_{1} \in[0, T]$ and by Lemma 5

$$
\begin{aligned}
& \mathbb{E}\left[\sup _{t \in\left[0, t_{1}\right]}\left|Q_{t \wedge v_{n}}\right|^{2}\right] \\
& \leq \mathbb{E}\left|Q_{0}\right|^{2}+\left(K_{1}+K_{2}+K_{3} C_{2}\right) \int_{0}^{t_{1} \wedge v_{n}} \mathbb{E}\left[\sup _{0 \leq s \leq t}\left|Q_{s}\right|^{2}\right] \mathrm{d} t \\
&+K_{3} C_{3} T+\lambda_{1} k^{2} C T \\
&+2 \mathbb{E}\left[\sup _{t \in\left[0, t_{1}\right]} \int_{0}^{t \wedge v_{n}}\left(Q_{s}, g\left(s, Z_{2}(s)\right) \mathrm{d} W_{s}\right)\right] \\
&+2 \mathbb{E}\left[\sup _{t \in\left[0, t_{1}\right]} \int_{0}^{t \wedge v_{n}}\left(Q_{s}, h\left(s, Z_{2}(s), \bar{\gamma}(s)\right)\right) \mathrm{d} \widetilde{N}_{s}\right] \\
&+\mathbb{E}\left[\sup _{t \in\left[0, t_{1}\right]} \int_{0}^{t \wedge v_{n}}\left|h\left(s, Z_{2}(s), \bar{\gamma}(s)\right)\right|^{2} \mathrm{~d} \widetilde{N}_{s}\right] .
\end{aligned}
$$

By Burkholder-Davis-Gundy inequality, we have

$$
\begin{aligned}
& 2 \mathbb{E}\left[\sup _{t \in\left[0, t_{1}\right]} \int_{0}^{t \wedge v_{n}}\left(Q_{s}, g\left(s, Z_{2}(s)\right) d W_{s}\right)\right] \\
& \leq \frac{1}{4} \mathbb{E}\left[\sup _{t \in\left[0, t_{1}\right]}\left|Q_{t}\right|^{2}\right]+K_{4} \int_{0}^{t_{1} \wedge v_{n}}\left\|g\left(t, Z_{2}(t)\right)\right\|_{2}^{2} \mathrm{~d} t
\end{aligned}
$$

$$
\begin{aligned}
& \leq \frac{1}{4} \mathbb{E}\left[\sup _{t \in\left[0, t_{1}\right]}\left|Q_{t}\right|^{2}\right]+K_{4} k^{2} \int_{0}^{t_{1} \wedge v_{n}} \mathbb{E}\left\|Z_{2}(t)\right\|^{2} \mathrm{~d} t \\
& \mathbb{E}\left[\sup _{t \in\left[0, t_{1}\right]} \int_{0}^{t \wedge v_{n}}\left|h\left(s, Z_{2}(s), \bar{\gamma}(s)\right)\right|^{2} \mathrm{~d} \widetilde{N}_{s}\right] \\
& \leq 4 \lambda_{1} \mathbb{E} \int_{0}^{t_{1} \wedge v_{n}}\left|h\left(t, Z_{2}(t), \bar{\gamma}(s)\right)\right|^{2} \mathrm{~d} t \\
& \leq K_{5} k^{2} \int_{0}^{t_{1} \wedge v_{n}} \mathbb{E}\left\|Z_{2}(t)\right\|^{2}+\mathbb{E}|\bar{\gamma}(s)-\gamma(s)|^{2} \mathrm{~d} t \\
& \leq K_{5} k^{2} \int_{0}^{t_{1} \wedge v_{n}} \mathbb{E}\left\|Z_{2}(t)\right\|^{2} \mathrm{~d} t+K_{5} k^{2} C T, \\
& 2 \mathbb{E}\left[\sup _{t \in\left[0, t_{1}\right]} \int_{0}^{t \wedge v_{n}}\left(Q_{s}, h\left(s, Z_{2}(s), \bar{\gamma}(s)\right)\right) \mathrm{d} \widetilde{N}_{s}\right] \\
& \leq \frac{1}{4} \mathbb{E}\left[\sup _{t \in\left[0, t_{1}\right]}\left|Q_{t}\right|^{2}\right]+K_{6} \int_{0}^{t_{1} \wedge v_{n}}\left|h\left(t, Z_{2}(t), \bar{\gamma}(s)\right)\right|^{2} \mathrm{~d} t \\
& \leq \frac{1}{4} \mathbb{E}\left[\sup _{t \in\left[0, t_{1}\right]}\left|Q_{t}\right|^{2}\right] \\
& +K_{6} k^{2} \int_{0}^{t_{1} \wedge v_{n}} \mathbb{E}\left\|Z_{2}(t)\right\|^{2} \mathrm{~d} t+K_{6} k^{2} C T,
\end{aligned}
$$

for some positive constants $K_{4}, K_{5}, K_{6}>0$. Substituting (33) into (32) and then by Lemma 5 , we can obtain that

$$
\begin{aligned}
& \mathbb{E}\left[\sup _{t \in\left[0, t_{1}\right]}\left|Q_{t \wedge v_{n}}\right|^{2}\right] \\
& \leq 2 \mathbb{E}\left|Q_{0}\right|^{2} \\
&+2\left(K_{1}+K_{2}+K_{3} C_{2}\right) \int_{0}^{t_{1} \wedge v_{n}} \mathbb{E}\left[\sup _{0 \leq s \leq t}\left|Q_{s}\right|^{2}\right] \mathrm{d} t \\
&+2 K_{3} C_{3} T+2\left(K_{4}+K_{5}+K_{6}\right) k^{2} \int_{0}^{t_{1} \wedge v_{n}} \mathbb{E}\left\|Z_{2}(t)\right\|^{2} \mathrm{~d} t \\
&+2\left(\lambda_{1}+K_{5}+K_{6}\right) k^{2} C T \\
& \leq 2\left[K_{1}+K_{2}+K_{3} C_{2}+\left(K_{4}+K_{5}+K_{6}\right) k^{2} C_{2}\right] \\
& \times \int_{0}^{t_{1} \wedge v_{n}} \mathbb{E}\left[\sup _{0 \leq s \leq t}\left|Q_{s}\right|^{2}\right] \mathrm{d} t \\
&+2 \mathbb{E}\left|Q_{0}\right|^{2}+2 K_{3} C_{3} T+2\left(K_{4}+K_{5}+K_{6}\right) k^{2} C_{3} T \\
&+2\left(\lambda_{1}+K_{5}+K_{6}\right) k^{2} C T \\
&:= K_{7}+K_{8} \int_{0}^{t_{1} \wedge v_{n}} \mathbb{E}\left[\sup _{0 \leq s \leq t}\left|Q_{s}\right|^{2}\right] \mathrm{d} t
\end{aligned}
$$


where

$$
\begin{aligned}
K_{7}= & 2 \mathbb{E}\left|Q_{0}\right|^{2}+2 K_{3} C_{3} T+2\left(K_{4}+K_{5}+K_{6}\right) k^{2} C_{3} T \\
& +2\left(\lambda_{1}+K_{5}+K_{6}\right) k^{2} C T, \\
K_{8}= & 2\left[K_{1}+K_{2}+K_{3} C_{2}+\left(K_{4}+K_{5}+K_{6}\right) k^{2} C_{2}\right] .
\end{aligned}
$$

Applying the continuous Gronwall inequality, we can easy get

$$
\mathbb{E}\left[\sup _{t \in[0, T]}\left|Q_{t \wedge v_{n}}\right|^{2}\right] \leq C_{4},
$$

with $C_{4}=K_{7} \exp \left(K_{8} T\right)$.

$$
\begin{aligned}
& \mathbb{E}\left|Q_{t}-Z_{1}(t)\right|^{2} \leq C_{5} \Delta, \\
& \mathbb{E}\left|Q_{t}-Z_{2}(t)\right|^{2} \leq C_{6} \Delta,
\end{aligned}
$$

where $Z_{1}(t), Z_{2}(t)$, and $Q_{t}$ are defined by (14), (15), and (17), respectively.

Proof. Consider $t \in[n \Delta,(n+1) \Delta] \subseteq[0, T]$; we have

$$
\begin{aligned}
Q_{t}-Z_{1}(t) \\
=Q_{t}-Q_{n} \\
=-\int_{n \Delta}^{t} \frac{\partial Q_{s}}{\partial a} \mathrm{~d} s \\
\quad+\int_{n \Delta}^{t}\left[(1-\theta) f\left(s, Z_{1}(s)\right)+\theta f\left(s, Z_{2}(s)\right)\right] \mathrm{d} s \\
\quad-\int_{n \Delta}^{t} \mu(s, a)\left[(1-\theta) Z_{1}(s)+\theta Z_{2}(s)\right] \mathrm{d} s \\
+\int_{n \Delta}^{t} g\left(s, Z_{2}(s)\right) \mathrm{d} W_{s}+\int_{n \Delta}^{t} h\left(s, Z_{2}(s), \bar{\gamma}(s)\right) \mathrm{d} N_{s} .
\end{aligned}
$$

Squaring both sides and using the element inequality $(a+b+c+e+f)^{2} \leq 5|a|^{2}+5|b|^{2}+5|c|^{2}+5|d|^{2}+5|e|^{2}$, we have

$$
\begin{aligned}
& \left|Q_{t}-Z_{1}(t)\right|^{2} \\
& \leq 5\left|\int_{n \Delta}^{t} \frac{\partial Q_{s}}{\partial a} \mathrm{~d} s\right|^{2} \\
& \quad+5\left|\int_{n \Delta}^{t}\left[(1-\theta) f\left(s, Z_{1}(s)\right)+\theta f\left(s, Z_{2}(s)\right)\right] \mathrm{d} s\right|^{2} \\
& \quad+5\left|\int_{n \Delta}^{t} \mu(s, a)\left[(1-\theta) Z_{1}(s)+\theta Z_{2}(s)\right] \mathrm{d} s\right|^{2} \\
& \quad+5\left|\int_{n \Delta}^{t} g\left(s, Z_{2}(s)\right) \mathrm{d} W(s)\right|^{2} \\
& \quad+5\left|\int_{n \Delta}^{t} h\left(s, Z_{2}(s), \bar{\gamma}(s)\right) \mathrm{d} N(s)\right|^{2}
\end{aligned}
$$

Now, the Cauchy-Schwarz inequality, condition ( $\mathbb{1} 3$ ), and the compensated Poisson process give

$$
\begin{aligned}
\left|Q_{t}-Z_{1}(t)\right|^{2} & \leq 5 \Delta \int_{n \Delta}^{t}\left|\frac{\partial Q_{s}}{\partial a}\right|^{2} \mathrm{~d} s \\
& +5 \bar{\mu}^{2} \Delta \int_{n \Delta}^{t}\left|(1-\theta) Z_{1}(s)+\theta Z_{2}(s)\right|^{2} \mathrm{~d} s \\
& +5 \Delta \int_{n \Delta}^{t}\left|(1-\theta) f\left(s, Z_{1}(s)\right)+\theta f\left(s, Z_{2}(s)\right)\right|^{2} \mathrm{~d} s \\
& +5\left|\int_{n \Delta}^{t} g\left(s, Z_{2}(s)\right) \mathrm{d} W(s)\right|^{2} \\
& +10\left|\int_{n \Delta}^{t} h\left(s, Z_{2}(s), \bar{\gamma}(s)\right) \mathrm{d} \widetilde{N}(s)\right|^{2} \\
& +10 \lambda_{1}^{2}\left|\int_{n \Delta}^{t} h\left(s, Z_{2}(s), \bar{\gamma}(s)\right) \mathrm{d} s\right|^{2} .
\end{aligned}
$$

Taking mathematical expectation, by element inequality $(a+b)^{2} \leq 2|a|^{2}+2|b|^{2}$, and using the martingale isometry, we have

$$
\begin{aligned}
\mathbb{E} \mid Q_{t}- & \left.Z_{1}(t)\right|^{2} \\
\leq & 5 \Delta \int_{n \Delta}^{t}\left|\frac{\partial Q_{s}}{\partial a}\right|^{2} \mathrm{~d} s \\
& +5 \bar{\mu}^{2} \Delta \int_{n \Delta}^{t} \mathbb{E}\left(\left|Z_{1}(s)\right|^{2}+\left|Z_{2}(s)\right|^{2}\right) \mathrm{d} s
\end{aligned}
$$




$$
\begin{aligned}
& +10 \Delta \int_{n \Delta}^{t} \mathbb{E}\left|f\left(s, Z_{1}(s)\right)\right|^{2}+\mathbb{E}\left|f\left(s, Z_{2}(s)\right)\right|^{2} \mathrm{~d} s \\
& +5 \int_{n \Delta}^{t} \mathbb{E}\left|g\left(s, Z_{2}(s)\right)\right|^{2} \mathrm{~d} s \\
& +10 \lambda_{1} \int_{n \Delta}^{t} \mathbb{E}\left|h\left(s, Z_{2}(s), \bar{\gamma}(s)\right)\right|^{2} \mathrm{~d} s \\
& +10 \lambda_{1}^{2} \Delta \int_{n \Delta}^{t} \mathbb{E}\left|h\left(s, Z_{2}(s), \bar{\gamma}(s)\right)\right|^{2} \mathrm{~d} s .
\end{aligned}
$$

By conditions (आ1) and (आ2), we get

$$
\begin{aligned}
\mathbb{E} \mid Q_{t}- & \left.Z_{1}(t)\right|^{2} \\
\leq & 5 \Delta \int_{n \Delta}^{t}\left|\frac{\partial Q_{s}}{\partial a}\right|^{2} \mathrm{~d} s \\
& +5 \bar{\mu}^{2} \Delta \int_{n \Delta}^{t} \mathbb{E}\left(\left|Z_{1}(s)\right|^{2}+\left|Z_{2}(s)\right|^{2}\right) \mathrm{d} s \\
& +10 \Delta k^{2} \int_{n \Delta}^{t} \mathbb{E}\left|Z_{1}(s)\right|^{2}+\mathbb{E}\left|Z_{2}(s)\right|^{2} \mathrm{~d} s \\
& +5 k^{2} \int_{n \Delta}^{t} \mathbb{E}\left|Z_{2}(s)\right|^{2} \mathrm{~d} s \\
& +\left(10 \lambda_{1}+10 \lambda_{1}^{2} \Delta\right) k^{2} \int_{n \Delta}^{t} \mathbb{E}\left|Z_{2}(s)\right|^{2} \\
= & 5 \Delta \int_{n \Delta}^{t}\left|\frac{\partial Q_{s}}{\partial a}\right|^{2} \mathrm{~d} s+\left(10 \Delta k^{2}+5 \bar{\mu}^{2} \Delta\right) \int_{n \Delta}^{t} \mathbb{E}\left|Z_{1}(s)\right|^{2} \mathrm{~d} s \\
& +\left(5+10 \lambda_{1}+10 \lambda_{1}^{2} \Delta\right) k^{2} \int_{n \Delta}^{t} \mathbb{E}\left|Z_{2}(s)\right|^{2} \mathrm{~d} s \\
& +\left(10 \lambda_{1}+10 \lambda_{1}^{2} \Delta\right) k^{2} C \Delta .
\end{aligned}
$$

Since $Z_{1}(t) \equiv Q_{n}$ and $Z_{2}(t) \equiv Q_{n}^{*}$ on $[n \Delta t,(n+1) \Delta t)$, we have

$$
\begin{aligned}
\mathbb{E} \mid Q_{t}- & \left.Z_{1}(t)\right|^{2} \\
\leq & 5 \Delta \int_{n \Delta}^{t}\left|\frac{\partial Q_{s}}{\partial a}\right|^{2} \mathrm{~d} s+\left(10 \Delta k^{2}+5 \bar{\mu}^{2} \Delta\right) \int_{n \Delta}^{t} \mathbb{E}\left|Q_{n}\right|^{2} \mathrm{~d} s \\
& +\left(5+10 \lambda_{1}+10 \lambda_{1}^{2} \Delta\right) k^{2} \int_{n \Delta}^{t} \mathbb{E}\left|Q_{n}^{*}\right|^{2} \mathrm{~d} s \\
& +\left(10 \lambda_{1}+10 \lambda_{1}^{2} \Delta\right) k^{2} C \Delta .
\end{aligned}
$$

Then by Lemmas 5 and 6, we can derive

$$
\begin{aligned}
\mathbb{E} \mid Q_{t}- & \left.Z_{1}(t)\right|^{2} \\
\leq & {\left[10 \Delta k^{2}+5 \bar{\mu}^{2} \Delta\right.} \\
& \left.+C_{2}\left(5+10 \lambda_{1}+10 \lambda_{1}^{2} \Delta\right) k^{2}\right] \int_{n \Delta}^{t} \mathbb{E}\left|Q_{n}\right|^{2} \mathrm{~d} s \\
& +\left(5+10 \lambda_{1}+10 \lambda_{1}^{2} \Delta\right) k^{2} C_{3} \Delta+5 \Delta \int_{n \Delta}^{t}\left|\frac{\partial Q_{s}}{\partial a}\right|^{2} \mathrm{~d} s \\
& +\left(10 \lambda_{1}+10 \lambda_{1}^{2} \Delta\right) k^{2} C \Delta \\
\leq & {\left[10 \Delta k^{2}+5 \bar{\mu}^{2} \Delta+C_{2}\left(5+10 \lambda_{1}+10 \lambda_{1}^{2} \Delta\right) k^{2}\right] C_{4} \Delta } \\
& +\left(5+10 \lambda_{1}+10 \lambda_{1}^{2} \Delta\right) k^{2} C_{3} \Delta+5 \Delta \int_{n \Delta}^{t}\left|\frac{\partial Q_{s}}{\partial a}\right|^{2} \mathrm{~d} s \\
& +\left(10 \lambda_{1}+10 \lambda_{1}^{2} \Delta\right) k^{2} C \Delta \leq C_{5} \Delta,
\end{aligned}
$$

where

$$
\begin{aligned}
C_{5}= & {\left[10 k^{2}+5 \bar{\mu}^{2}+C_{2}\left(5+10 \lambda_{1}+10 \lambda_{1}^{2}\right) k^{2}\right] C_{4} } \\
& +\left(10 \lambda_{1}+10 \lambda_{1}^{2} \Delta\right) k^{2} C+\left(5+10 \lambda_{1}+10 \lambda_{1}^{2}\right) k^{2} C_{3} \\
& +5 \int_{n \Delta}^{t}\left|\frac{\partial Q_{s}}{\partial a}\right|^{2} \mathrm{~d} s .
\end{aligned}
$$

Thus we can prove (37), and a similar analysis gives the proof of (38).

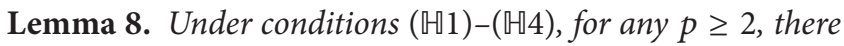
exists a positive constant $C_{7}$ such that

$$
\mathbb{E}\left[\sup _{t \in[0, T]}\left|Q_{t}\right|^{p}\right] \leq C_{7}
$$

Proof. The proof is similar to that of Lemma 4.

\section{Main Result}

Now we use the above lemmas to prove a strong convergent result.

Theorem 9. Under condition $(\mathbb{H} 1)-(\mathbb{M} 4)$, let $0<\theta<1,0<$ $\Delta<\min \left\{1,1 / \theta(k+\bar{\mu}), 1 / 2 \sqrt{3\left(\bar{\mu}^{2}+k^{2}\right)}\right\}$; then there exists a constant $C_{8}>0$ such that

$$
\mathbb{E}\left[\sup _{0 \leq t \leq T}\left|K_{t \wedge v_{n}}-Q_{t \wedge v_{n}}\right|^{2}\right] \leq C_{8} \Delta^{1-(1 / q)} .
$$


Proof. From (2) and (17), we have

$$
\begin{aligned}
K_{t}-Q_{t} & \\
= & -\int_{0}^{t} \frac{\partial\left(K_{s}-Q_{s}\right)}{\partial a} \mathrm{~d} s \\
& -\int_{0}^{t} \mu(s, a)\left[(1-\theta)\left(K_{s}-Z_{1}(s)\right)\right. \\
& \left.+\theta\left(K_{s}-Z_{2}(s)\right)\right] \mathrm{d} s \\
& +\int_{0}^{t}\left[(1-\theta)\left(f\left(s, K_{s}\right)-f\left(s, Z_{1}(s)\right)\right)\right. \\
& +\int_{0}^{t}\left[g\left(s, K_{s}\right)-g\left(s, Z_{2}(s)\right)\right] \mathrm{d} W_{s} \\
& +\int_{0}^{t}\left[h\left(s, K_{s}, \gamma(s)\right)-h\left(s, Z_{2}(s), \bar{\gamma}(s)\right)\right] \mathrm{d} N_{s} .
\end{aligned}
$$

Using the generalized Itô formula it yields

$$
\begin{aligned}
& \left|K_{t}-Q_{t}\right|^{2} \\
& =2 \int_{0}^{t}\left\langle K_{s}-Q_{s},-\frac{\partial\left(K_{s}-Q_{s}\right)}{\partial a}\right\rangle \mathrm{d} s \\
& -2 \int_{0}^{t}\left(K_{s}-Q_{s}, \mu(s, a)\right. \\
& \left.\times\left[(1-\theta)\left(K_{s}-Z_{1}(s)\right)+\theta\left(K_{s}-Z_{2}(s)\right)\right] \mathrm{d} s\right) \\
& +\int_{0}^{t}\left(K_{s}-Q_{s},\left[(1-\theta)\left(f\left(s, K_{s}\right)-f\left(s, Z_{1}(s)\right)\right)\right.\right. \\
& \left.\left.+\theta\left(f\left(s, P_{s}\right)-f\left(s, Z_{2}(s)\right)\right)\right] \mathrm{d} s\right) \\
& +\int_{0}^{t}\left\|g\left(s, K_{s}\right)-g\left(s, Z_{2}(s)\right)\right\|_{2}^{2} \mathrm{~d} s \\
& +2 \int_{0}^{t}\left(K_{s}-Q_{s},\left[g\left(s, K_{s}\right)-g\left(s, Z_{2}(s)\right)\right] d W_{s}\right) \\
& +2 \int_{0}^{t}\left(K_{s}-Q_{s}, h\left(s, K_{s}, \gamma(s)\right)\right. \\
& \left.-h\left(s, Z_{2}(s), \bar{\gamma}(s)\right)\right) \mathrm{d} \widetilde{N}_{s} \\
& +2 \lambda_{1} \int_{0}^{t}\left(K_{s}-Q_{s}, h\left(s, K_{s}, \gamma(s)\right)\right. \\
& \left.-h\left(s, Z_{2}(s), \bar{\gamma}(s)\right)\right) \mathrm{d} s \\
& +\int_{0}^{t}\left|h\left(s, K_{s}, \gamma(s)\right)-h\left(s, Z_{2}(s), \bar{\gamma}(s)\right)\right|^{2} \mathrm{~d} \widetilde{N}_{s} \\
& +\lambda_{1} \int_{0}^{t}\left|h\left(s, K_{s}, \gamma(s)\right)-h\left(s, Z_{2}(s), \bar{\gamma}(s)\right)\right|^{2} \mathrm{~d} s .
\end{aligned}
$$

By Cauchy-Schwartz inequality and condition (ㄴ22), we have

$$
\begin{aligned}
\left|K_{t}-Q_{t}\right|^{2} & \leq \\
\leq & \left.A^{2} F_{1}^{2} \eta^{2}+\mu_{0}+\lambda_{1}+1\right) \int_{0}^{t}\left|K_{s}-Q_{s}\right|^{2} \mathrm{~d} s \\
& +2\left(K^{2}+\mu_{0}\right) \int_{0}^{t}\left|K_{s}-Z_{1}(s)\right|^{2} \mathrm{~d} s \\
& +2 \lambda_{1} K^{2} \int_{0}^{t}|\gamma(s)-\bar{\gamma}(s)|^{2} \mathrm{~d} s \\
& +\left(3 K^{2}+2 \lambda_{1} K^{2}+2 \mu_{0}\right) \int_{0}^{t}\left|K_{s}-Z_{2}(s)\right|^{2} \mathrm{~d} s \\
& +2 \int_{0}^{t}\left(K_{s}-Q_{s},\left[g\left(s, K_{s}\right)-g\left(s, Z_{2}(s)\right)\right] \mathrm{d} W_{s}\right) \\
& +2 \int_{0}^{t}\left(K_{s}-Q_{s}, h\left(s, K_{s}, \gamma(s)\right)\right. \\
& +\int_{0}^{t}\left|h\left(s, K_{s}, \gamma(s)\right)-h\left(s, Z_{2}(s), \bar{\gamma}(s)\right)\right|^{2} \mathrm{~d} \widetilde{N}_{s} .
\end{aligned}
$$

Hence for any $t \in[0, T]$

$$
\begin{aligned}
& \mathbb{E} \sup _{s \in[0, t]}\left|K_{s \wedge v_{n}}-Q_{s \wedge v_{n}}\right|^{2} \\
& \leq\left(A^{2} F_{1}^{2} \eta^{2}+\mu_{0}+\lambda_{1}+1\right) \\
& \times \int_{0}^{t \wedge v_{n}} \mathbb{E} \sup _{r \in[0, s]}\left|K_{r \wedge v_{n}}-Q_{r \wedge v_{n}}\right|^{2} \mathrm{~d} s \\
& +2\left(k^{2}+\mu_{0}\right) \int_{0}^{t \wedge v_{n}} \mathbb{E}\left\|K_{s}-Z_{1}(s)\right\|_{c}^{2} \mathrm{~d} s \\
& +2 \lambda_{1} k^{2} C \Delta^{1-(1 / q)} \\
& +\left(3 k^{2}+2 \lambda_{1} k^{2}+2 \mu_{0}\right) \int_{0}^{t \wedge v_{n}} \mathbb{E}\left\|K_{s}-Z_{1}(s)\right\|_{c}^{2} \mathrm{~d} s \\
& +2 \mathbb{E} \sup _{s \in[0, t]} \int_{0}^{s \wedge v_{n}}\left(K_{r}-Q_{r},\left[g\left(r, K_{r}\right)\right.\right. \\
& \left.\left.-g\left(r, Z_{2}(r)\right)\right] \mathrm{d} W_{r}\right) \\
& +2 \mathbb{E} \sup _{s \in[0, t]} \int_{0}^{s \wedge v_{n}}\left(K_{r}-Q_{r}, h\left(r, K_{r}, \gamma(r)\right)\right. \\
& \left.-h\left(s, Z_{2}(r), \bar{\gamma}(r)\right)\right) \mathrm{d} \widetilde{N}_{r} \\
& +\mathbb{E} \sup _{s \in[0, t]} \int_{0}^{s \wedge v_{n}} \mid h\left(r, K_{r}, \gamma(r)\right) \\
& -\left.h\left(r, Z_{2}(r), \bar{\gamma}(r)\right)\right|^{2} \mathrm{~d} \widetilde{N}_{r} .
\end{aligned}
$$


10

Abstract and Applied Analysis

Now using the Burkholder-Davis-Gundy inequality and Lemma 2, we have

$$
\begin{aligned}
& 2 \mathbb{E}\left[\sup _{s \in[0, t]} \int_{0}^{s \wedge v_{n}}\left(K_{r}-Q_{r},\left[g\left(r, K_{r}\right)-g\left(r, Z_{2}(r)\right)\right] \mathrm{d} W_{r}\right)\right] \\
& \leq 8 \mathbb{E}\left[\sup _{s \in[0, t]}\left|K_{s \wedge v_{n}}-Q_{s \wedge v_{n}}\right|\right. \\
& \left.\times\left(\int_{0}^{t \wedge v_{n}}\left\|g\left(s, K_{s}\right)-g\left(t, Z_{2}(s)\right)\right\|_{2}^{2} \mathrm{~d} s\right)^{1 / 2}\right] \\
& \leq \frac{1}{4} \mathbb{E}\left[\sup _{s \in[0, t]}\left|K_{s \wedge v_{n}}-Q_{s \wedge v_{n}}\right|^{2}\right] \\
& +k_{1} \int_{0}^{t \wedge v_{n}} \mathbb{E}\left\|K_{s}-Z_{2}(s)\right\|^{2} \mathrm{~d} s, \\
& 2 \mathbb{E}\left[\sup _{s \in[0, t]} \int_{0}^{s \wedge v_{n}}\left(K_{r}-Q_{r},\left(h\left(r, K_{r}\right)-h\left(s, Z_{2}(r)\right)\right)\right) \mathrm{d} \widetilde{N}_{r}\right] \\
& \leq 8 \lambda_{1} \mathbb{E}\left[\sup _{s \in[0, t]}\left|K_{s \wedge v_{n}}-Q_{s \wedge v_{n}}\right|\right. \\
& \times\left(\int_{0}^{t \wedge v_{n}} \mid h\left(s, K_{s}, \gamma(s)\right)\right. \\
& \left.\left.-\left.h\left(s, Z_{2}(s), \bar{\gamma}(s)\right)\right|^{2} \mathrm{~d} s\right)^{1 / 2}\right] \\
& \leq \frac{1}{4} \mathbb{E}\left[\sup _{s \in[0, t]}\left|K_{s \wedge v_{n}}-Q_{s \wedge v_{n}}\right|^{2}\right] \\
& +k_{2} \int_{0}^{t \wedge v_{n}} \mathbb{E}\left\|K_{s}-Z_{2}(s)\right\|^{2}+\mathbb{E}|\gamma(s)-\bar{\gamma}(s)|^{2} \mathrm{~d} s \\
& \leq \frac{1}{4} \mathbb{E}\left[\sup _{s \in[0, t]}\left|K_{s \wedge v_{n}}-Q_{s \wedge v_{n}}\right|^{2}\right] \\
& +k_{2} \int_{0}^{t \wedge v_{n}} \mathbb{E}\left\|K_{s}-Z_{2}(s)\right\|^{2} \mathrm{~d} s+k_{2} C \Delta^{1-(1 / q)}, \\
& \mathbb{E}\left[\sup _{s \in[0, t]} \int_{0}^{s \wedge v_{n}}\left|h\left(r, K_{r}, \gamma(r)\right)-h\left(r, Z_{2}(r), \bar{\gamma}(r)\right)\right|^{2} \mathrm{~d} \widetilde{N}_{r}\right] \\
& \leq 4 \lambda_{1} \int_{0}^{t \wedge v_{n}} \mathbb{E}\left|h\left(s, K_{s}, \gamma(s)\right)-h\left(s, Z_{2}(s), \bar{\gamma}(s)\right)\right|^{2} \mathrm{~d} s \\
& \leq 4 \lambda_{1} k^{2} \int_{0}^{t \wedge v_{n}} \mathbb{E}\left|K_{s}-Z_{2}(s)\right|^{2}+\mathbb{E}|\gamma(s)-\bar{\gamma}(s)|^{2} \mathrm{~d} s \\
& \leq k_{3} \int_{0}^{t \wedge v_{n}} \mathbb{E}\left\|K_{s}-Z_{2}(s)\right\|^{2} \mathrm{~d} s+k_{3} C \Delta^{1-(1 / q)} \text {, }
\end{aligned}
$$

where $k_{1}, k_{2}, k_{3}$ are positive constants. Therefore, inserting (53) into (52) it has

$$
\begin{aligned}
\mathbb{E} \sup _{s \in[0, t]}\left|K_{s \wedge v_{n}}-Q_{s \wedge v_{n}}\right|^{2} \\
\leq\left(A^{2} F_{1}^{2} \eta^{2}+\mu_{0}+\lambda_{1}+1\right)
\end{aligned}
$$

$$
\begin{aligned}
& \times \int_{0}^{t \wedge v_{n}} \mathbb{E} \sup _{r \in[0, s]}\left|K_{r \wedge v_{n}}-Q_{r \wedge v_{n}}\right|^{2} \mathrm{~d} s \\
& +2\left(k^{2}+\mu_{0}\right) \int_{0}^{t \wedge v_{n}} \mathbb{E}\left\|K_{s}-Z_{1}(s)\right\|_{c}^{2} \mathrm{~d} s \\
& +2 \lambda_{1} k^{2} C \Delta^{1-(1 / q)} \\
& +\left(3 k^{2}+2 \lambda_{1} k^{2}+2 \mu_{0}\right) \int_{0}^{t \wedge v_{n}} \mathbb{E}\left\|K_{s}-Z_{2}(s)\right\|_{c}^{2} \mathrm{~d} s \\
& +\left(k_{1}+k_{2}+k_{3}\right) \int_{0}^{t \wedge v_{n}} \mathbb{E}\left\|K_{s}-Q_{s}\right\|_{c}^{2} \mathrm{~d} s \\
& +\left(k_{2}+k_{3}\right) C \Delta^{1-(1 / q)}+\frac{1}{2} \mathbb{E}\left[\sup _{s \in[0, t]}\left|P_{s \wedge v_{n}}-Q_{s \wedge v_{n}}\right|^{2}\right] .
\end{aligned}
$$

Then using Lemma 7, we have

$$
\begin{aligned}
& \underset{s \in[0, t]}{\mathbb{E} \sup _{s \wedge v_{n}}}\left|Q_{s \wedge v_{n}}\right|^{2} \\
& \leq\left(A^{2} F_{1}^{2} \eta^{2}+\mu_{0}+\lambda_{1}+1\right) \\
& \times \int_{0}^{t \wedge v_{n}} \mathbb{E} \sup _{r \in[0, s]}\left|P_{r \wedge v_{n}}-Q_{r \wedge v_{n}}\right|^{2} \mathrm{~d} s \\
&+ 4\left(k^{2}+\mu_{0}\right) \int_{0}^{t \wedge v_{n}} \mathbb{E}\left\|P_{s}-Q_{s}\right\|_{c}^{2} \mathrm{~d} s \\
&+ 4\left(k^{2}+\mu_{0}\right) C_{5} \Delta \\
&+ 2\left(3 k^{2}+2 \lambda_{1} k^{2}+2 \mu_{0}+k_{1}+k_{2}+k_{3}\right) \\
& \times \int_{0}^{t \wedge v_{n}} \mathbb{E}_{0}\left\|P_{s}-Q_{s}\right\|_{c}^{2} \mathrm{~d} s \\
&+ 2\left(3 k^{2}+2 \lambda_{1} k^{2}+2 \mu_{0}+k_{1}+k_{2}+k_{3}\right) C_{6} \Delta \\
&+\left(2 \lambda_{1} k^{2}+k_{2}+k_{3}\right) C \Delta^{1-(1 / q)} \\
&+ \frac{1}{2}\left[\mathbb{E}\left[\sup _{s \in[0, t]}\left|P_{s \wedge v_{n}}-Q_{s \wedge v_{n}}\right|^{2}\right] .\right.
\end{aligned}
$$

That is,

$$
\begin{aligned}
\underset{s \in[0, t]}{\mathbb{E} \sup _{s \wedge v_{n}}}\left|P_{s \wedge v_{n}}\right|^{2} \\
\leq 2\left(A^{2} F_{1}^{2} \eta^{2}+\mu_{0}+\lambda_{1}+1\right) \\
\quad \times \int_{0}^{t \wedge v_{n}} \mathbb{E} \sup _{r \in[0, s]}\left|P_{r \wedge v_{n}}-Q_{r \wedge v_{n}}\right|^{2} \mathrm{~d} s \\
+8\left(k^{2}+\mu_{0}\right) \int_{0}^{t \wedge v_{n}} \mathbb{E} \sup _{r \in[0, s]}\left|P_{r \wedge v_{n}}-Q_{r \wedge v_{n}}\right|^{2} \mathrm{~d} s
\end{aligned}
$$




$$
\begin{aligned}
& +4\left(3 k^{2}+2 \lambda_{1} K^{2}+2 \mu_{0}+k_{1}+k_{2}+k_{3}\right) \\
& \quad \times \int_{0}^{t \wedge v_{n}} \mathbb{E} \sup _{r \in[0, s]}\left|P_{r \wedge v_{n}}-Q_{r \wedge v_{n}}\right|^{2} \mathrm{~d} s \\
& +8\left(k^{2}+\mu_{0}\right) C_{5} \Delta \\
& +4\left(3 k^{2}+2 \lambda_{1} k^{2}+2 \mu_{0}+k_{1}+k_{2}+k_{3}\right) C_{6} \Delta \\
& +2\left(2 \lambda_{1} k^{2}+k_{2}+k_{3}\right) C \Delta^{1-(1 / q)} \\
& \leq d_{1} \Delta^{1-(1 / q)}+d_{2} \int_{0}^{t} \mathbb{E} \sup _{r \in[0, s]}\left|P_{r \wedge v_{n}}-Q_{r \wedge v_{n}}\right|^{2} \mathrm{~d} s,
\end{aligned}
$$

where

$$
\begin{aligned}
d_{1}= & 8\left(k^{2}+\mu_{0}\right) C_{5} \\
& +4\left(3 k^{2}+2 \lambda_{1} k^{2}+2 \mu_{0}+k_{1}+k_{2}+k_{3}\right) C_{6} \\
& +2\left(2 \lambda_{1} k^{2}+k_{2}+k_{3}\right) C \Delta^{1-(1 / q)}, \\
d_{2}= & 2\left(A^{2} F_{1}^{2} \eta^{2}+\mu_{0}+\lambda_{1}+1\right)+8\left(k^{2}+\mu_{0}\right) \\
& +4\left(3 k^{2}+2 \lambda_{1} k^{2}+2 \mu_{0}+k_{1}+k_{2}+k_{3}\right) .
\end{aligned}
$$

Using the Gronwall inequality, we have

$$
\mathbb{E} \sup _{s \in[0, t]}\left|P_{s \wedge v_{n}}-Q_{s \wedge v_{n}}\right|^{2} \leq d_{1} \exp \left(d_{2} T\right) \Delta:=C_{8} \Delta^{1-(1 / q)} .
$$

Since for any $t \in[0, T]$, thus we have

$$
\mathbb{E} \sup _{t \in[0, T]}\left|P_{t \wedge v_{n}}-Q_{t \wedge v_{n}}\right|^{2} \leq C_{8} \Delta^{1-(1 / q)}
$$

The proof is completed.

Theorem 10. Under conditions $(\mathbb{H} 1)-(\mathbb{M} 4)$, let $0<\theta<1,0<$ $\Delta<\min \left\{1,1 / \theta(k+\bar{\mu}), 1 / 2 \sqrt{3\left(\bar{\mu}^{2}+k^{2}\right)}\right\}$; then there exists a constant $C_{9}>0$ such that

$$
\mathbb{E}\left[\sup _{0 \leq t \leq T}\left|K_{t}-Q_{t}\right|^{2}\right] \leq C_{9} \Delta t+o(\Delta) .
$$

Proof. Let $e(t)=K_{t}-Q_{t}$; it is easy to see that

$$
\begin{gathered}
\mathbb{E}\left[\sup _{0 \leq t \leq T}|e(t)|^{2}\right] \\
=\mathbb{E}\left[\sup _{0 \leq t \leq T}|e(t)|^{2} I_{\left\{\tau_{n}>T, \sigma_{n}>T\right\}}\right] \\
+\mathbb{E}\left[\sup _{0 \leq t \leq T}|e(t)|^{2} I_{\left\{\tau_{n} \leq T\right.} \text { or } \sigma_{n} \leq T\right\}
\end{gathered}
$$

$$
\begin{aligned}
= & \mathbb{E}\left[\sup _{0 \leq t \leq T}|e(t)|^{2} I_{\left\{v_{n}>T\right\}}\right] \\
& +\mathbb{E}\left[\sup _{0 \leq t \leq T}|e(t)|^{2} I_{\left\{\tau_{n} \leq T \text { or } \sigma_{n} \leq T\right\}}\right] \\
\leq & \mathbb{E}\left[\sup _{0 \leq t \leq T}\left|e\left(t \wedge v_{n}\right)\right|^{2}\right] \\
& +\mathbb{E}\left[\sup _{0 \leq t \leq T}|e(t)|^{2} I_{\left\{\tau_{n} \leq T \text { or } \sigma_{n} \leq T\right\}}\right] .
\end{aligned}
$$

Recall the Young inequality: for $(1 / p)+(1 / q)=1(p, q>0)$, we have

$$
\begin{aligned}
a b & \leq a \delta^{1 / p} \frac{b}{\delta^{1 / p}} \\
& \leq \frac{\left(a \delta^{1 / p}\right)^{p}}{p}+\frac{b^{q}}{q \delta^{q / p}}=\frac{a^{p} \delta}{p}+\frac{b^{q}}{q \delta^{q / p}}, \quad \forall a, b, \delta>0 .
\end{aligned}
$$

Let $p=2, \delta=\Delta$; we have

$$
\begin{aligned}
& \mathbb{E}\left[\sup _{0 \leq t \leq T}|e(t)|^{2} I_{\left\{\tau_{n} \leq T\right.} \text { or } \sigma_{n} \leq T\right\} \\
& \quad \leq \frac{\Delta}{2} \mathbb{E}\left[\sup _{0 \leq t \leq T}|e(t)|^{4}\right]+\frac{1}{2 \Delta} P\left\{\tau_{n} \leq T \text { or } \sigma_{n} \leq T\right\} .
\end{aligned}
$$

Note

$$
\begin{aligned}
\mathbb{E}\left[\sup _{0 \leq t \leq T}|e(t)|^{4}\right] & \leq 8\left(\mathbb{E}\left[\sup _{0 \leq t \leq T}\left|P_{t}\right|^{4}\right]+\mathbb{E}\left[\sup _{0 \leq t \leq T}\left|Q_{t}\right|^{4}\right]\right) \\
& \leq 8\left(C_{1}+C_{7}\right) .
\end{aligned}
$$

By Lemma 4, then

$$
P\left\{\tau_{n} \leq T\right\}=\mathbb{E}\left[I_{\left\{\tau_{n} \leq T\right\}} \frac{\left|P_{\tau_{n}}\right|^{p}}{n^{4}}\right] \leq \frac{1}{n^{4}} \mathbb{E}\left[\sup _{0 \leq t \leq T}\left|P_{t}\right|^{4}\right] \leq \frac{C_{1}}{n^{4}} ;
$$

similarly, the result can be derived for $\sigma_{n}$

$$
P\left\{\sigma_{n} \leq T\right\}=\mathbb{E}\left[I_{\left\{\tau_{n} \leq T\right\}} \frac{\left|Q_{\tau_{n}}\right|^{p}}{n^{4}}\right] \leq \frac{1}{n^{4}} \mathbb{E}\left[\sup _{0 \leq t \leq T}\left|Q_{t}\right|^{4}\right] \leq \frac{C_{7}}{n^{4}},
$$

so that

$$
\begin{aligned}
P\left\{\sigma_{d} \leq T \text { or } \nu_{d} \leq T\right\} & \leq P\left\{\sigma_{d} \leq T\right\}+P\left\{\nu_{d} \leq T\right\} \\
& \leq \frac{\left(C_{1}+C_{7}\right)}{n^{4}}
\end{aligned}
$$




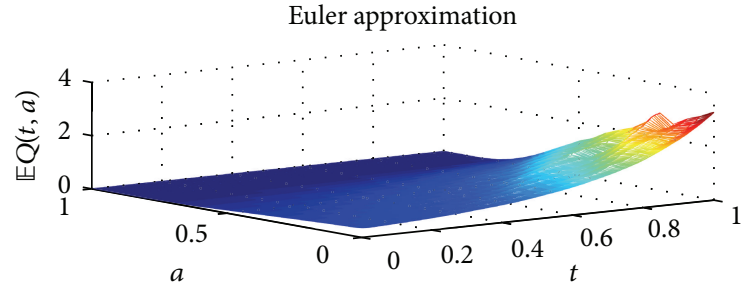

(a)

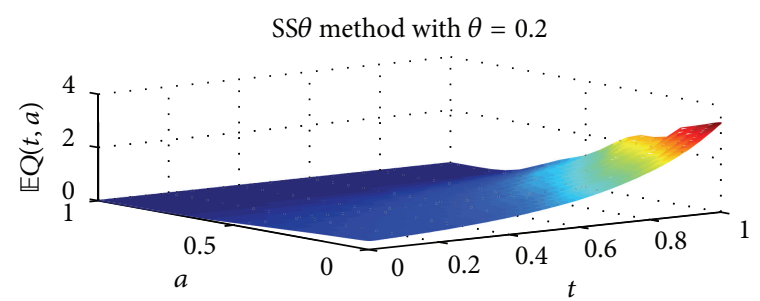

(b)

FIGURE 1: Expectation of numerical solution with 1000 tests, where $\Delta=0.005$ and $\Delta a=0.05$.

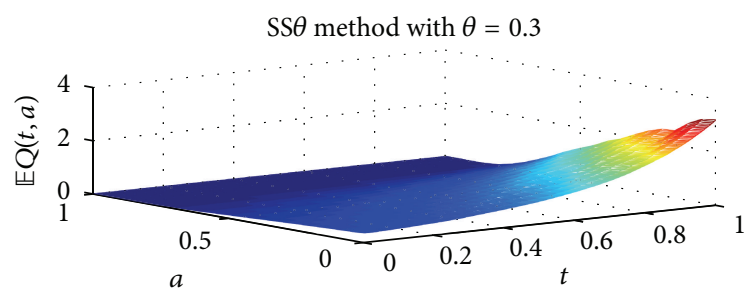

(a)

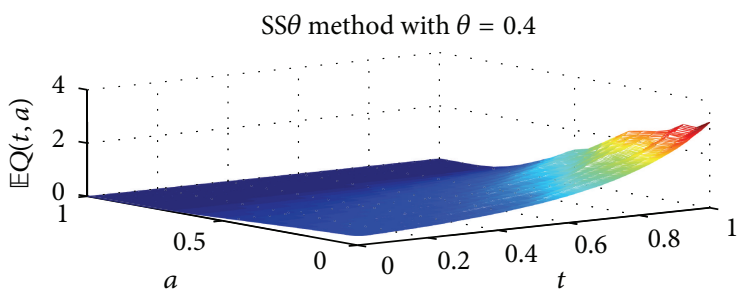

(b)

FIGURE 2: Expectation of numerical solution with 1000 tests, where $\Delta=0.005$ and $\Delta a=0.05$.

Then

$$
\left.\mathbb{E}\left[\sup _{0 \leq t \leq T}|e(t)|^{2} I_{\left\{\tau_{n} \leq T\right.} \text { or } \sigma_{n} \leq T\right\}\right] \leq 4 \Delta\left(C_{1}+C_{7}\right)+\frac{\left(C_{1}+C_{7}\right)}{2 \Delta n^{4}} .
$$

By Theorem 9, (61) becomes

$$
\mathbb{E}\left[\sup _{0 \leq t \leq T}|e(t)|^{2}\right] \leq C_{8} \Delta+o(\Delta)+4 \Delta\left(C_{1}+C_{7}\right)+\frac{\left(C_{1}+C_{7}\right)}{2 \Delta n^{4}}
$$

Let $n \geq 2\left(\Delta^{2}\right)^{-1 / 4}$; then

$$
\begin{aligned}
\mathbb{E}\left[\sup _{0 \leq t \leq T}|e(t)|^{2}\right] \\
\quad \leq C_{8} \Delta+o(\Delta)+4 \Delta\left(C_{1}+C_{7}\right)+\Delta\left(C_{1}+C_{7}\right) \\
:=C_{9} \Delta+o(\Delta),
\end{aligned}
$$

where $C_{9}=5\left(C_{1}+C_{7}\right)+C_{8}$. The proof is completed.

Theorem 11. Under the conditions $(\mathbb{H} 1)-(\mathbb{4} 4)$ there exists a constant $C$ such that $\mathbb{E}\left[\left|\gamma_{i}\right|^{2 q}\right] \leq C$ for some $q>1$, the numerical approximate solution (1) will converge to the exact solution to (1) in the sense

$$
\lim _{\Delta \rightarrow 0} \mathbb{E}\left[\sup _{0 \leq t \leq T}\left|K_{t}-Q_{t}\right|^{2}\right]=0
$$

Proof. The proof is easily deduced from Theorem 10.

\section{Numerical Example}

In this section, we consider the following stochastic agedependent capital system with random jump magnitudes agedependent in [10]

$$
\begin{gathered}
\mathrm{d} K(t, a)=-\frac{1}{(1-a)^{2}} K \mathrm{~d} t+2 t K \mathrm{~d} t-t K \mathrm{~d} W(t) \\
+\bar{\gamma}(t) K \mathrm{~d} N(t), \quad \text { in } D, \\
K(t, 0)=\frac{t^{2}}{(1-t)^{2}} \int_{0}^{1} K(t, a) \mathrm{d} a, \quad \text { in } t \in[0, T], \\
K(0, a)=e^{-1 /(1-a)}, \quad \text { in } a \in(0, A), \\
\mathbb{N}(t)=\int_{0}^{1} K(t, a) \mathrm{d} a, \quad \text { in } t \in[0, T],
\end{gathered}
$$

where $W(t)$ is a scalar Brownian motion and $N(t)$ is a scalar Poisson process with intensity $\lambda_{1}=1$. Let $\bar{\gamma}(t)$ be defined in (16). Take $T=1, A=1$, and $\bar{\gamma}$ to be a lognormal random variable such that $\mathbb{E}[\bar{\gamma}]=0.2$ and $\mathbb{E}\left[\bar{\gamma}^{2}\right]=0.25$.

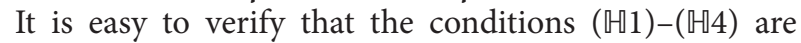
satisfied. Then the approximate solution will converge to the true solution of (1) for any $(t, a) \in(0,1) \times(0,1)$ in the sense of Theorem 11. Obviously, $K(t, a)$ in (72) cannot be solved explicitly. It is necessary to know numerical approximation $Q(t, a)$ of $K(t, a)$.

Now we fix step sizes $\Delta=0.005$ and $\Delta a=0.05$ and change the parameter $\theta$ in all figures. Then we compare the expectation of the numerical solution, where $\mathbb{E}[Q(t, a)]=$ $(1 / 1000) \sum_{n=1}^{1000} Q_{n}(t, a)$.

In Figure 1, we show the expectation of numerical solution of the system (72) by Euler method in [10] and splitstep $\theta$-method with $\theta=0.2$, respectively. From the figure, 


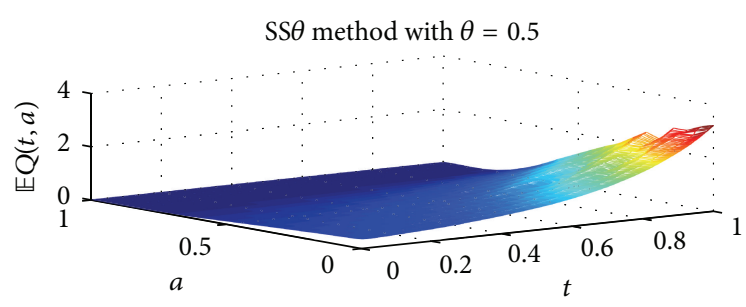

(a)

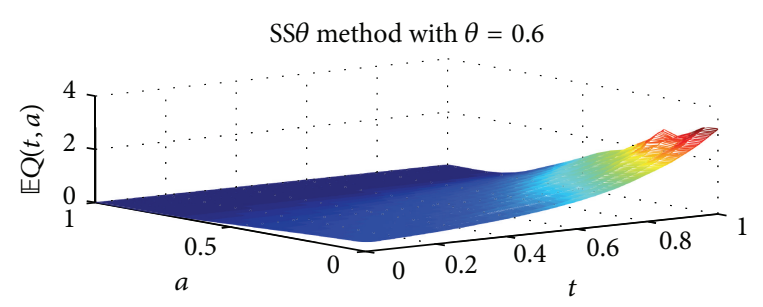

(b)

FIGURE 3: Expectation of numerical solution with 1000 tests, where $\Delta=0.005$ and $\Delta a=0.05$.

we can see that the split-step $\theta$-method also reveals the agedependent capital system tendency.

In Figures 2 and 3, the four pictures are simulation for numerical solution $Q_{n}(t, a)$ of the system (72) by split-step $\theta$ method with $\theta=0.3,0.4,0.5$, and 0.6 , respectively.

\section{Conclusion}

Due to the complexity of this model, the comparison between the Euler approximation and split-step methods is not possible in this paper. Similarly, error analysis is also not obtained in this paper due to unavailability of closed form solutions. They will be discussed in the future researches. But the visualization of Figures 1, 2, and 3 provides the information about the split-step methods which coincide with the Euler approximation. The comparison between Euler approximation and split-step methods in the stochastic differential equations in [11-13] shows that the split-step methods are better than the Euler approximation. Similarly, we believe that this new approximation of the system (1) in this paper is a good approximation when compared with the Euler approximation.

\section{Conflict of Interests}

The authors declare that there is no conflict of interests regarding the publication of this paper.

\section{Acknowledgments}

This research was supported with funds provided by the National Natural Science Foundation of China (nos. 11226321, 11272229 , and 11102132). The second author is working under the research project named as UGC Research Award 20122014 of University Grants Commission, Bahadur Shah Zafar Marg, New Delhi-110002 with award number F.30-1/2013(SAII)/RA-2012-14-OB-TAM-1324.

\section{References}

[1] Z. Wang, "Stability of solution to a class of investment system," Applied Mathematics and Computation, vol. 207, no. 2, pp. 340$345,2009$.

[2] Q.-M. Zhang, W.-K. Pang, and P.-K. Leung, "Exponential stability of numerical solutions for a class of stochastic age-dependent capital system with Poisson jumps," Journal of Computational and Applied Mathematics, vol. 235, no. 12, pp. 3369-3377, 2011.

[3] Q. M. Zhang, "Convergence of numerical solutions for a class stochastic age-dependent capital system with Markovian switching," Economic Modelling, vol. 28, pp. 1195-1201, 2011.

[4] R. Li, W. K. Pang, and Q. Wang, "Numerical analysis for stochastic age-dependent population equations with Poisson jumps," Journal of Mathematical Analysis and Applications, vol. 327, no. 2, pp. 1214-1224, 2007.

[5] L. Wang and X. Wang, "Convergence of the semi-implicit Euler method for stochastic age-dependent population equations with Poisson jumps," Applied Mathematical Modelling, vol. 34, no. 8, pp. 2034-2043, 2010.

[6] A. Rathinasamy, B. Yin, and B. Yasodha, "Numerical analysis for stochastic age-dependent population equations with Poisson jump and phase semi-Markovian switching," Communications in Nonlinear Science and Numerical Simulation, vol. 16, no. 1, pp. 350-362, 2011.

[7] D. J. Higham, X. Mao, and A. M. Stuart, "Exponential meansquare stability of numerical solutions to stochastic differential equations," LMS Journal of Computation and Mathematics, vol. 6, pp. 297-313, 2003.

[8] P. Glasserman and N. Merener, "Convergence of a discretization scheme for jump-diffusion processes with state-dependent intensities," Proceedings of The Royal Society of London A, vol. 460, no. 2041, pp. 111-127, 2004.

[9] A. Svishchuk and A. Kalemanova, "The stochastic stability of interest rates with jump changes," Theory of Probability and Mathematical Statistics, vol. 61, pp. 161-172, 2000.

[10] Q. Zhang and A. Rathinasamy, "Convergence of numerical solutions for a class of stochastic age-dependent capital system with random jump magnitudes," Applied Mathematics and Computation, vol. 219, no. 14, pp. 7297-7305, 2013.

[11] D. J. Higham and P. E. Kloeden, "Numerical methods for nonlinear stochastic differential equations with jumps," Numerische Mathematik, vol. 101, no. 1, pp. 101-119, 2005.

[12] J. Tan and H. Wang, "Convergence and stability of the splitstep backward Euler method for linear stochastic delay integrodifferential equations," Mathematical and Computer Modelling, vol. 51, no. 5-6, pp. 504-515, 2010.

[13] X. Ding, Q. Ma, and L. Zhang, "Convergence and stability of the split-step $\theta$-method for stochastic differential equations," Computers \& Mathematics with Applications, vol. 60, no. 5, pp. 1310-1321, 2010.

[14] A. Rathinasamy, "Split-step $\theta$-methods for stochastic agedependent population equations with Markovian switching," Nonlinear Analysis. Real World Applications, vol. 13, no. 3, pp. 1334-1345, 2012. 
[15] Q. M. Zhang, W. A. Liu, and Z. K. Nie, "Existence, uniqueness and exponential stability for stochastic age-dependent population," Applied Mathematics and Computation, vol. 154, no. 1, pp. 183-201, 2004.

[16] G. D. Chalmers and D. J. Higham, "Convergence and stability analysis for implicit simulations of stochastic differential equations with random jump magnitudes," Discrete and Continuous Dynamical Systems B, vol. 9, no. 1, pp. 47-64, 2008.

[17] M. Wei, "Convergence analysis of semi-implicit Euler methods for solving stochastic equations with variable delays and random jump magnitudes," Journal of Computational and Applied Mathematics, vol. 235, no. 8, pp. 2569-2580, 2011.

[18] D. R. Smart, Fixed Point Theorems, Cambridge University Press, London, UK, 1974.

[19] A. Gardoń, "The order of approximations for solutions of Itôtype stochastic differential equations with jumps," Stochastic Analysis and Applications, vol. 22, no. 3, pp. 679-699, 2004. 


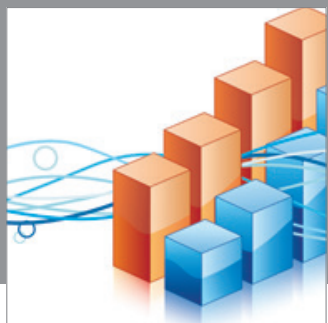

Advances in

Operations Research

mansans

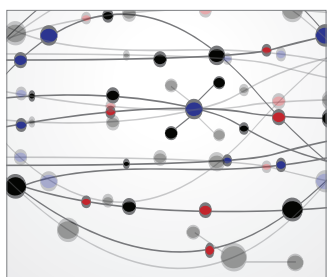

The Scientific World Journal
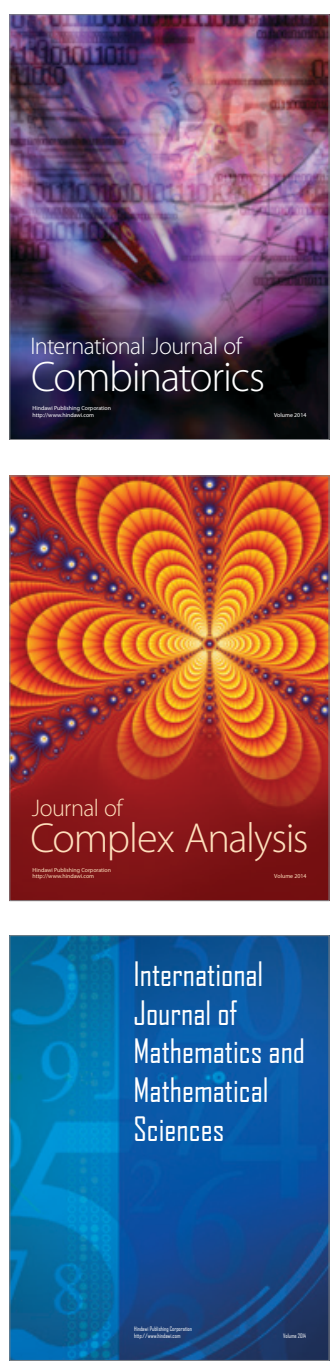
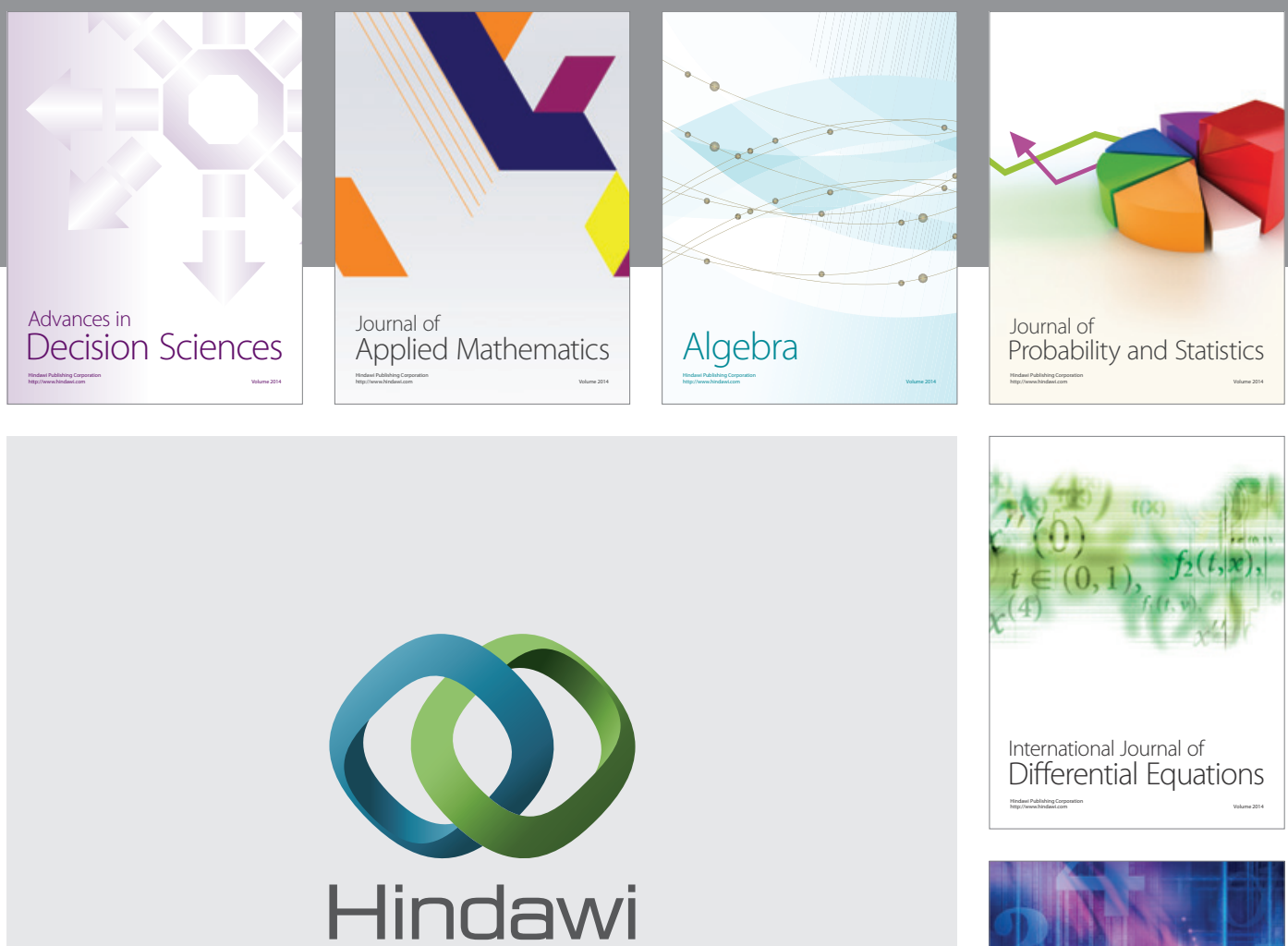

Submit your manuscripts at http://www.hindawi.com
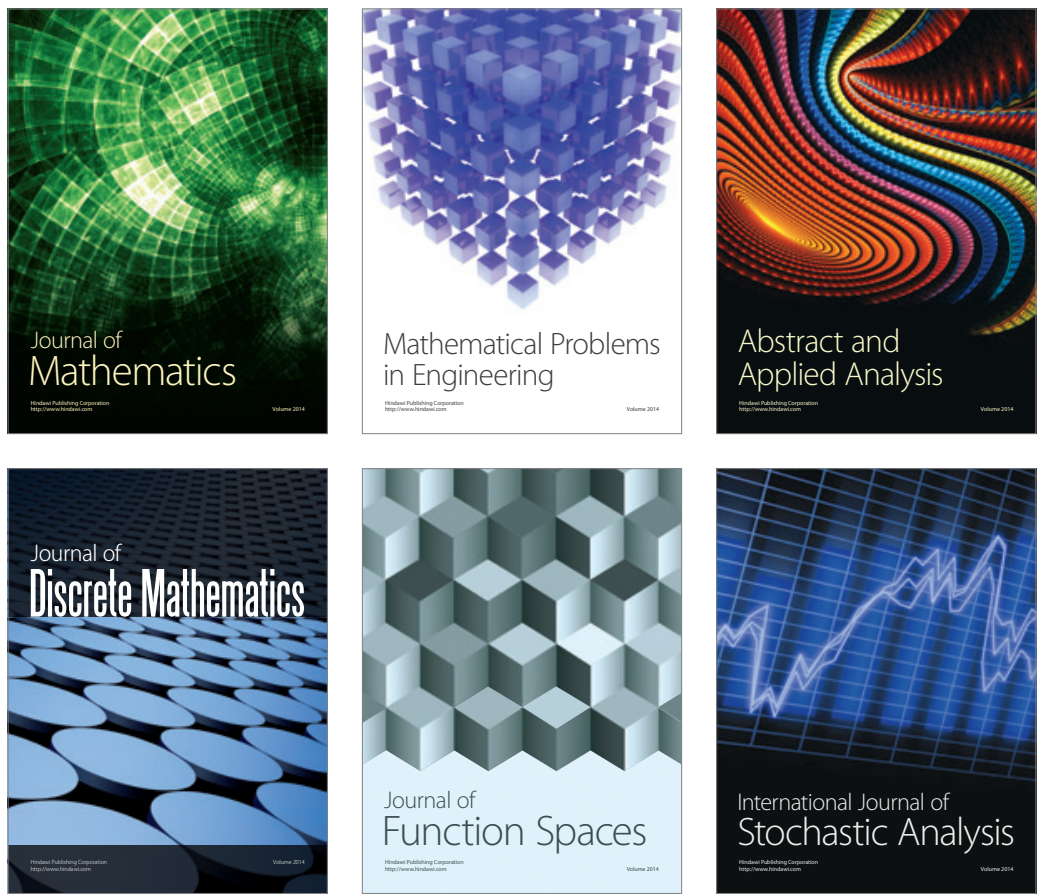

Journal of

Function Spaces

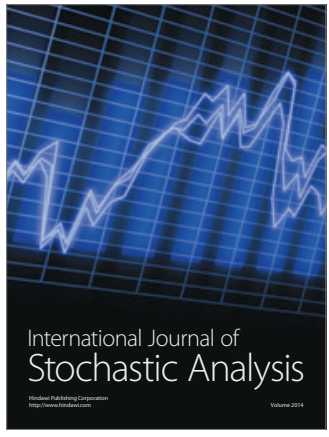

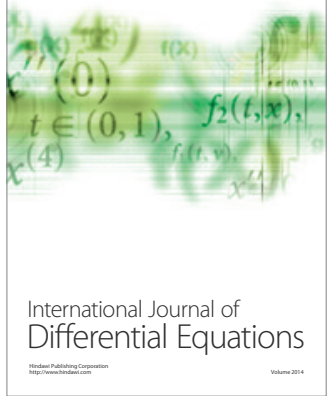
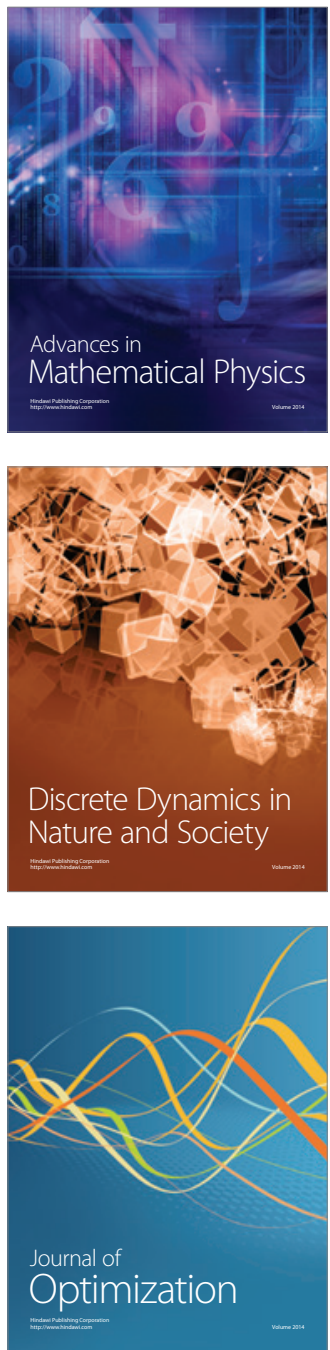